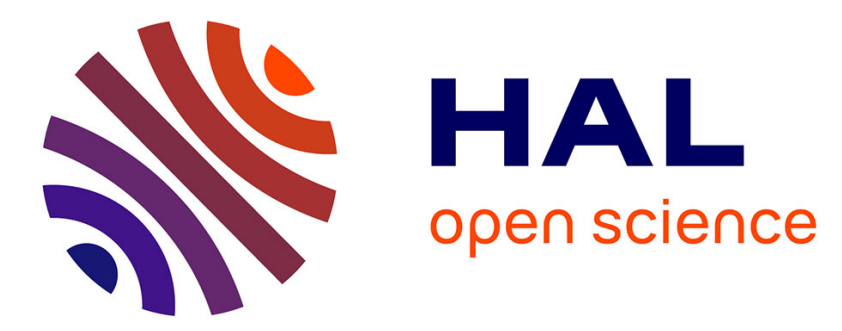

\title{
Analysis of necking based on a one-dimensional model
}

\author{
Basile Audoly, John W. Hutchinson
}

\section{To cite this version:}

Basile Audoly, John W. Hutchinson. Analysis of necking based on a one-dimensional model. Journal of the Mechanics and Physics of Solids, 2016, 97 (68). hal-01212041

\section{HAL Id: hal-01212041 \\ https://hal.science/hal-01212041}

Submitted on 6 Oct 2015

HAL is a multi-disciplinary open access archive for the deposit and dissemination of scientific research documents, whether they are published or not. The documents may come from teaching and research institutions in France or abroad, or from public or private research centers.
L'archive ouverte pluridisciplinaire HAL, est destinée au dépôt et à la diffusion de documents scientifiques de niveau recherche, publiés ou non, émanant des établissements d'enseignement et de recherche français ou étrangers, des laboratoires publics ou privés. 


\title{
Analysis of necking based on a one-dimensional model
}

\author{
Basile Audoly ${ }^{\mathrm{a}, \mathrm{b}}$, John W. Hutchinson ${ }^{\mathrm{c}}$ \\ ${ }^{a}$ Sorbonne Universités, UPMC Univ Paris 06, UMR 7190, Institut Jean Le Rond d'Alembert, F-75005, Paris, France \\ ${ }^{b}$ CNRS, UMR 7190, Institut Jean Le Rond d'Alembert, F-75005, Paris, France \\ ${ }^{c}$ School of Engineering and Applied Sciences, Harvard University, Cambridge, MA 02138, USA
}

\begin{abstract}
Dimensional reduction is applied to derive a one-dimensional energy functional governing tensile necking localization in a family of initially uniform prismatic solids, including as particular cases rectilinear blocks in plane strain and cylindrical bars undergoing axisymmetric deformations. The energy functional depends on both the axial stretch and its gradient. The coefficient of the gradient term is derived in an exact and general form. The one-dimensional model is used to analyze necking localization for nonlinear elastic materials that experience a maximum load under tensile loading, and for a class of nonlinear materials that mimic elastic-plastic materials by displaying a linear incremental response when stretch switches from increasing to decreasing. Bifurcation predictions for the onset of necking from the simplified theory compared with exact results suggest the approach is highly accurate at least when the departures from uniformity are not too large. Post-bifurcation behavior is analyzed to the point where the neck is fully developed and localized to a region on the order of the thickness of the block or bar. Applications to the nonlinear elastic and elastic-plastic materials reveal the highly unstable nature of necking for the former and the stable behavior for the latter, except for geometries where the length of the block or bar is very large compared to its thickness. A formula for the effective stress reduction at the center of a neck is established based on the one-dimensional model, which is similar to that suggested by Bridgman (1952).
\end{abstract}

Keywords: B. Beams and columns, B. Elastic-plastic material, C. Stability and bifurcation, C. Asymptotic analysis

\section{Introduction with précis of the dimensional reduction}

The tension test is one of the canonical methods to measure the stress-strain behavior of materials. A well-known complication for ductile materials which undergo a maximum load in the test is necking wherein deformation localizes in a region on the order of the specimen thickness at some location along the length of the specimen. Considère (1885) described the connection of the onset of the localization process to the maximum load, and the criteria for the stress and strain at the onset of necking bear his name. Since Considère's time, necking has received a great deal of attention in the scientific and engineering literatures not only because of its importance in measuring material properties (Bridgman, 1952) but because it is often a precursor to material failure (Marciniak and Kuczyński, 1967). Necking phenomena are highly nonlinear owing to both material and geometric nonlinearities. Detailed fully nonlinear analyses of necking emerged in the early 1970's after finite strain plasticity formulations were developed and computers became available with the capacity to carry out the requisite computations. Chen's (1971) Galerkin-like approach to necking in bars was soon followed by finite element analyses of necking in round bars by Needleman (1972) and Norris et al. (1978). These and subsequent studies provided the neck shape and the stress and strain distributions within the neck for cross-sections that had localized to less than one half of the cross-sectional area outside of the neck.

During this same period, analytical conditions for the onset of necking were derived within the framework of Hill's (1961) bifurcation theory elastic-plastic solids: both the cases of a cylinder having a circular cross-section (Hutchinson and Miles, 1974) and of a 2D rectangular block in plane strain (Hill and Hutchinson, 1975) were considered. These analyses were later extended in two ways: Scherzinger and Triantafyllidis 
(1998) considered the linear stability of general prismatic solids (with non-circular cross-section), Triantafyllidis et al. (2007) addressed the post-bifurcation problem by means of a Lyapunov-Schmidt-Koiter expansion for a $2 \mathrm{D}$ rectangular block for various constitutive laws. In line with these local stability results, Sivaloganathan and Spector (2011) showed that the homogeneous solution is, in a global sense, the only absolute minimizer of the energy for a class of constitutive behaviors such that the load/displacement curve does not possess a maximum. The latter approach tells nothing about the necking instability; the other 2D or 3D approaches are limited to the linear or weakly non-linear analysis of necking.

Approximate 1D models offer an appealing alternative to 2D and 3D approaches, making it possible to analyze the essential nonlinear aspects of necking. Ericksen (1975) notes that simple bar models, whereby the density of stored elastic energy is a function of the centerline stretch only, give rise to discontinuous solutions past the point of maximum load. A regularized elastic energy functional is therefore needed, which is typically assumed to depend on the gradient of the centerline stretch as well. Based on regularized models of this kind, Antman (1973) carries out a linear bifurcation analysis, Antman and Carbone (1977) address the initial post-bifurcation behavior; Coleman and Newman (1988) construct non-linear solutions exhibiting localized necks, while Owen (1987) discusses their stability.

With the exception of the work of Mielke, discussed below, one-dimensional models used for the analysis of necking are not derived from 2D or 3D continuum mechanics by proper dimensional reduction. The model of Owen (1987), for instance, is based on a kinematical assumption for the shear which is not applicable in the slender limit, as noted by Mielke (1991); the dimensional reduction of Coleman and Newman (1988) is based on a similar assumption and we discuss it later in detail. To the best of our knowledge, the dimensional reduction of Mielke (1991), which uses the central manifold theorem, is the only one which is asymptotically exact, i.e. it predicts not only the form of the regularized energy functional but also the correct value of its coefficients. Mielke's analysis is done for a $2 \mathrm{D}$ rectangular block made of a hyperelastic material. Here, we extend his work in the following ways: we consider 3D prismatic solids having an arbitrary cross-section and made of an orthotropic material, and we recover the case of a 2D block made of an isotropic material as a special case; we also derive a simple formula for the modulus associated with the regularizing secondgradient term, which captures the dependence on both the cross-sectional geometry and on the constitutive law; finally, we extend the 1D model to account for the elastic unloading of elastic/plastic materials.

The analytical details of the dimensional reduction leading to the one-dimensional necking model are presented in Section 2 for a family of uniform prismatic solids. The final mathematical model is simple even though the reduction itself is somewhat technical. Thus, a summary of the equations governing the 1D model is included in this Introduction, see below: this allows the reader to skip Section 2 and proceed directly to the applications covered in Section 3 and 4. In Section 3, the 1D model is used to characterize the response of a nonlinear elastic material whose loading curve displays a maximum load. In Section 4, we extend the model to account for the elastic unloading of an elastic/plastic material, and show that this specific unloading response can completely modify the global necking behavior. It should be emphasized that the $1 \mathrm{D}$ reduction is not meant to capture the transition from necking to shear localization, nor the shear localization itself, which often occurs in the advanced stages of necking in of metal bars or sheets.

The general derivation in Section 2 considers prismatic solids having an arbitrary cross-section and takes into account orthotropy and material compressibility, but in this section and in the applications attention is limited to $2 \mathrm{D}$ blocks or circular cylinders (round bars) and to isotropic nonlinear elastic materials that are incompressible. The geometry in the undeformed and deformed states is indicated in figure 1, including notation used throughout the paper. In the undeformed state, the block is rectangular with thickness $h$ and length $2 L$ in $2 \mathrm{D}$, and the round bar in $3 \mathrm{D}$ is uniform with a cross-section diameter $D$ and length $2 L$. The coordinate $X$ identifies material cross-sections in the undeformed state. In the deformed state, the cross-sections become curved and the coordinate of their average axial position is denoted by $x=\Lambda(X)$. The stretch is then defined as $\lambda(X)=\frac{\mathrm{d} \Lambda}{\mathrm{d} X}$; it characterizes the deformation state of the block or bar in the one-dimensional reduction. The lateral faces of the block (or the lateral surface of the bar) are traction-free. Particular attention will be given to solutions that are symmetric with respect to the point $X=x=0$, which is assumed to lie at the center of the neck. Idealized conditions are applied on the endpoints, namely symmetry conditions at $X=0$, and the condition of zero shear tractions at $X=L$. These boundary conditions admit the uniform stretch, $\lambda_{0}=1+\delta / L$ as one solution for an initially perfect block or bar, 
where $\delta$ is the elongation of the right-hand side half of the specimen.

For non-uniform stretching the dimensional reduction in Section 2 starts by observing that a nonhomogeneous configuration having planar cross-sections is close to (but not exactly at) equilibrium, and seeks an equilibrium solution by a perturbation method. This approach works under the assumption that the prescribed inhomogeneous stretch $\lambda(X)$ varies on a much longer length-scale than the cross-section dimension $h$ or $D$ : a linearized elasticity problem with small residual strain yields the sought equilibrium solution, which has curved cross-sections. The primary independent variable in the one-dimensional theory is the macroscopic stretch measure, $\lambda(X)$, and the dimensional reduction assigns an energy to any prescribed stretch distribution $\lambda(X)$.

We stress that the dimensional reduction does not start by linearizing near a uniform solution with constant stretch $\lambda(X)=\lambda_{0}$-if that were the case, its validity would be limited to configurations such that $\lambda(X)$ stays uniformly close to a constant value $\lambda_{0}$. Instead, we linearize near a non-equilibrium configuration having a slowly varying stretch $\lambda(X)$ - for a slender block or bar, the stretch $\lambda(X)$ may well vary significantly over the entire length. This feature is particularly desirable in the context of necking, where the relevant solutions are slowly variable, but far from homogeneous as a whole.

First, consider the block in plane strain, with thickness $h$ and out-of-plane stretch $\lambda_{I I}=1$. With the largest principal stretch as $\lambda_{I}=\lambda$ and the minimum principal stretch as $\lambda_{I I I}=1 / \lambda_{I}$, the material is characterized microscopically by an energy density function $w_{0}\left(\lambda_{I}\right)$. The Cauchy (true) stress for a block that undergoes uniform stretch $\lambda_{0}=1+\delta / L$ is $\sigma_{11} \equiv \sigma_{0}\left(\lambda_{0}\right)=\lambda_{0} \frac{\mathrm{d} w_{0}}{\mathrm{~d} \lambda}\left(\lambda_{0}\right)$, while the nominal, or engineering stress, is $n_{0}\left(\lambda_{0}\right)=\frac{\mathrm{d} w_{0}}{\mathrm{~d} \lambda}\left(\lambda_{0}\right)$. The analysis in Section 2 provides the following result for the location of a material point in the deformed state $(x, y)$ in terms of its location in the undeformed state $(X, Y)$ :

$$
x=\Lambda(X)+\frac{1}{2 \lambda^{4}(X)} \frac{\mathrm{d} \lambda}{\mathrm{d} X}\left(Y^{2}-\frac{h^{2}}{12}\right) \quad \text { and } \quad y=\frac{1}{\lambda} Y .
$$

Here, $\Lambda(X)=\int_{0}^{X} \lambda\left(X^{\prime}\right) \mathrm{d} X^{\prime}$ denotes the mapping from the coordinate $X$ of a cross-section in reference configuration, to its average coordinate $\Lambda(X)$ in deformed configuration. The quantity $\lambda(X)$, defined as the derivative of $\Lambda(X)$, is also the cross-sectional average of the axial deformation gradient: we shall refer to it as the macroscopic stretch, or simply the stretch. By equation $(1.1), \lambda(X)$ is also the axial stretch of the two material filaments with equation $Y= \pm h /(2 \sqrt{3})$, initially parallel to the axis. The strain energy in the block (per unit depth out-of-plane) associated with this approximation is

$$
W=h \int_{0}^{L}\left[w_{0}(\lambda)+\frac{h^{2}}{2} b(\lambda)\left(\frac{\mathrm{d} \lambda}{\mathrm{d} X}\right)^{2}\right] \mathrm{d} X \quad \text { with } \quad b(\lambda)=\frac{n_{0}(\lambda)}{12 \lambda^{5}} \quad \text { (incompr. 2D block). }
$$

The second term in the integrand depends on the second gradient of displacement, the stretch $\lambda$ being its first gradient. In the modulus $b$ associated with this second-gradient term, note that the engineering stress for the homogeneous solutions $n_{0}(\lambda)=\frac{\mathrm{d} w_{0}}{\mathrm{~d} \lambda}$ is evaluated with the local value of the stretch $\lambda=\lambda(X)$. As a result, any nonlinearity present in the original $3 \mathrm{D}$ constitutive law is accurately captured in the $1 \mathrm{D}$ energy $W$ : this warrants the accuracy of the model into the nonlinear range.

The corresponding results for the round bar undergoing axisymmetric deformation are also expressed in terms of the stretch $\lambda(X)$. Denote the strain energy density for an isotropic incompressible elastic material under uniaxial stretch states $\left(\lambda_{I}=\lambda\right.$ and $\left.\lambda_{I I}=\lambda_{I I I}=\mu=\lambda_{I}^{-1 / 2}\right)$ by $w_{0}\left(\lambda_{I}\right)$. The true axial stresses under a uniform stretch, $\lambda_{0}$, is now $\sigma_{0}\left(\lambda_{0}\right)=\lambda_{0}^{2} \frac{\mathrm{d} w_{0}}{\mathrm{~d} \lambda}\left(\lambda_{0}\right)$, while the nominal stress is unchanged, $n_{0}\left(\lambda_{0}\right)=\frac{\mathrm{d} w_{0}}{\mathrm{~d} \lambda}\left(\lambda_{0}\right)$. Let $R$ denote the radial distance of a material point from the centerline in the undeformed state and $r$ denote this distance in the deformed state. In the one-dimension approximation, the location of a material point $(x, r)$ in the deformed state in terms of $(X, R)$ is

$$
x=\Lambda(X)+\frac{1}{4 \lambda^{3}(X)} \frac{\mathrm{d} \lambda}{\mathrm{d} X}\left(R^{2}-\frac{D^{2}}{8}\right) \quad \text { and } \quad r=\frac{1}{\sqrt{\lambda}} R,
$$

with $D$ as the diameter of the undeformed bar. The macroscopic stretch $\lambda(X)$ is still the cross-sectional average of the axial deformation gradient; for a a round bar, it also matches the microscopic stretch of the 

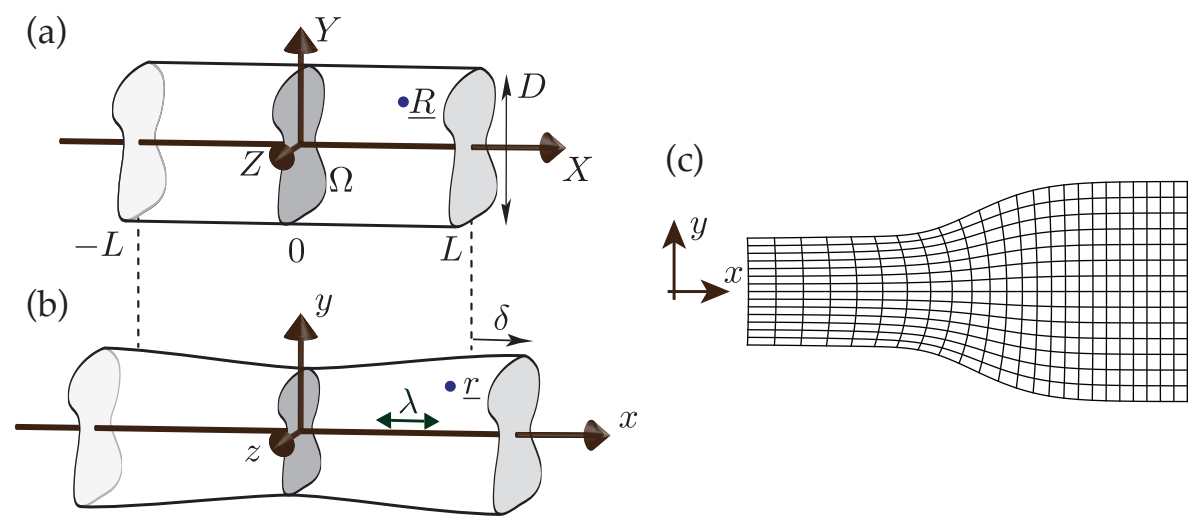

Figure 1: Dimensional reduction for a slender hyperelastic prismatic solid: (a) reference configuration, (b) actual configuration. (c) Sketch of microscopic displacement obtained by dimensional reduction, see equations (1.1) and (1.3): the cross-sections are curved and remain everywhere perpendicular to the material filaments initially parallel to the axis. This warrants in particular the cancellation of the shear stress tangent to the free surface.

filaments initially located at a distance $R=D /(2 \sqrt{2})$ from the axis, see the equation above. The energy in the bar is

$$
W=\left(\frac{\pi D^{2}}{4}\right) \int_{0}^{L}\left[w_{0}(\lambda)+\frac{D^{2}}{2} b(\lambda)\left(\frac{\mathrm{d} \lambda}{\mathrm{d} X}\right)^{2}\right] \mathrm{d} X \quad \text { with } \quad b(\lambda)=\frac{n_{0}(\lambda)}{32 \lambda^{4}} \quad \text { (incompr. circular cyl.). }
$$

\section{Dimensional reduction}

The goal of this section is to establish by means of asymptotic expansions the second-gradient bar model outlined above, which describes the onset and development of localization in a prismatic beam. We work with an hyperelastic material which is orthotropic and has its material symmetry axis aligned with the axis of the prismatic domain (note that his includes isotropic materials as a particular case). The material is assumed compressible; the incompressible limit will be worked out at the end as a special case. The cross-section geometry is arbitrary as well for the moment: the case of a round bar, or of a $2 \mathrm{D}$ block will also be worked out as a special case at the end, see $\S 2.9$. This section 2 can be skipped as it is independent of the rest of the paper, except for the final expressions of the second-gradient model which are summarized in $§ 2.8$. The models applicable to the special cases of a $2 \mathrm{D}$ block or a round bar made of an incompressible material have also been announced at the end of the Introduction.

\subsection{Geometry: a prismatic solid}

We consider an elastic body whose reference configuration is a prism of length $2 L$, having a typical cross-sectional diameter $D$, see figure 1 . We assume that the slenderness parameter $\epsilon=\frac{L}{D}$ is small, $\epsilon \ll 1$. We use a Cartesian frame $(X, Y, Z)$ in reference configuration, such that the axis $X$ is aligned with the axis of the prism, $(Y, Z)$ spans the cross-section. In addition, we take the origin $O$ of the frame to be the centroid of the reference cross-section $X=0$. Then, the axis $(O X)$ passes through the centroids of all cross-sections. The Cartesian basis is denoted by $\left(\underline{e}_{x}, \underline{e}_{y}, \underline{e}_{z}\right)$.

Let $\Omega$ be the domain of a cross-section, $(Y, Z) \in \Omega: D=\epsilon L$ is the typical diameter of $\Omega$. We define stretched coordinates $(\bar{Y}, \bar{Z})=\left(\frac{Y}{\epsilon}, \frac{Z}{\epsilon}\right)$ and a stretched cross-sectional domain $\bar{\Omega}=\frac{1}{\epsilon} \Omega$, such that $(\bar{Y}, \bar{Z}) \in \bar{\Omega}$. By construction, the stretched domain $\bar{\Omega}$ is independent of $\epsilon$ in the limit $\epsilon \rightarrow 0$, i.e. has a typical diameter of order 1.

We denote by $(x, y, z)$ the coordinates in current configuration. The transformation which maps a point $\underline{R}=(X, Y, Z)$ in reference configuration to a point $\underline{r}=(x, y, z)$ in actual configuration is specified by the 
functions $x(X, Y, Z), y(X, Y, Z)$ and $z(X, Y, Z)$. We denote by $x=\Lambda(X)$ the axial coordinate of the centroid of the deformed cross-section labeled by $X$ :

$$
\Lambda(x)=\langle x\rangle(X),
$$

where $\langle f\rangle(X)$ denotes the average of a quantity $f(X, Y, Z)$ over a cross-section, i.e. with respect to the transverse coordinates $Y$ and $Z$. This $\Lambda(X)$ is the primary kinematical variable of the one-dimensional model.

\subsection{Finite elasticity of a prismatic orthotropic solid}

Let $\underline{\underline{F}}$ denote the deformation gradient, $\underline{F}=\underline{\nabla r}(\underline{R})$, and $\underline{C}$ the Cauchy-Green strain tensor, $\underline{C}=\underline{F^{T}} \cdot \underline{\underline{F}}$, and the Green-St-Venant strain tensor, $\underline{\underline{e}}=(\underline{\underline{\underline{C}}}-\underline{\underline{1}}) / 2$. We consider a general hyperelastic orthotropic material, associated with the strain energy $E_{\mathrm{el}}$,

$$
E_{\mathrm{el}}=\int_{-L}^{L}\left(\iint_{\Omega} w(\underline{\underline{e}}) \mathrm{d} Y \mathrm{~d} Z\right) \mathrm{d} X
$$

where $w(\underline{\underline{e}})$ is the strain energy density. As stated in the first paragraph of $\S 2$, the axis of orthotropy is assumed to be aligned with the axis $X$ of the prismatic domain.

The first and second variations of the strain energy with respect to the strain $\underline{\underline{e}}$ define the second PiolaKirchhoff stress tensor $\underline{\underline{S}}(\underline{\underline{e}})$ and the tangent stiffness $\underline{\underline{\underline{\underline{A}}}}(\underline{\underline{e}})$, respectively. As a result the energy can be expanded about a configuration $\underline{\underline{e}}_{0}$ as

$$
w\left(\underline{\underline{e}}_{0}+\underline{\underline{\tilde{e}}}\right)=w\left(\underline{\underline{e}}_{0}\right)+\underline{\underline{S}}\left(\underline{\underline{e}}_{0}\right): \underline{\underline{\tilde{e}}}+\frac{1}{2} \underline{\underline{\tilde{e}}}: \underline{\underline{\underline{\underline{A}}}}\left(\underline{\underline{e}}_{0}\right): \underline{\underline{\tilde{e}}}+\cdots
$$

In the dimensional reduction, we will choose $\underline{\underline{e}}_{0}$ to be the simple traction solution, and treat any deviation from this state as a perturbation.

\subsection{Homogeneous solutions: uniform simple traction}

To start with, let us consider solutions describing a uniform stretching of the prismatic orthotropic solid along its axis, typically by end forces. The deformation gradient is then homogeneous and equibiaxial, $\underline{\underline{F}}=\underline{\underline{F}}$, and the transformation is affine, $\underline{x}=\underline{\underline{F}}_{0} \cdot \underline{X}$.

All quantities pertaining to this homogeneous equibiaxial solution will be labeled with a subscript ' 0 '. Let us denote by $\mu_{0}(\lambda)$ the transverse stretch as a function of the axial stretch $\lambda$ :

$$
\underline{\underline{F}}_{0}(\lambda)=\left(\begin{array}{ccc}
\lambda & 0 & 0 \\
0 & \mu_{0}(\lambda) & 0 \\
0 & 0 & \mu_{0}(\lambda)
\end{array}\right), \quad \underline{e}_{0}(\lambda)=\frac{1}{2}\left(\begin{array}{ccc}
\lambda^{2}-1 & 0 & 0 \\
0 & \mu_{0}^{2}(\lambda)-1 & 0 \\
0 & 0 & \mu_{0}^{2}(\lambda)-1
\end{array}\right) .
$$

Given the strain energy density for a compressible material, $w$, the function $\mu_{0}(\lambda)$ can be found by canceling the transverse stress. We consider the case of a stretched bar, i.e. we assume

$$
\lambda \geq 1 \geq \mu_{0}(\lambda)>0 .
$$

In terms of $\mu_{0}(\lambda)$, we can define the tangent (or incremental) Poisson's ratio $\nu_{0}(\lambda)$ as follows. For an increment of axial stretch from $\lambda$ to $\lambda+\mathrm{d} \lambda$, the transverse stretch varies from $\mu_{0}(\lambda)$ to $\mu_{0}(\lambda)+\mu_{0}^{\prime}(\lambda) \mathrm{d} \lambda$. The tangent Poisson's modulus is then defined as the ratio of the relative (Eulerian) variation of stretch in the transverse and axial directions:

$$
\nu_{0}(\lambda)=\left(\frac{-\mu_{0}^{\prime} \mathrm{d} \lambda}{\mu_{0}}\right) /\left(\frac{\mathrm{d} \lambda}{\lambda}\right)=-\frac{\lambda \mu_{0}^{\prime}(\lambda)}{\mu_{0}(\lambda)}=-\frac{\mathrm{d} \ln \mu_{0}}{\mathrm{~d} \ln \lambda} .
$$


For an incompressible material in dimension $3, \mu_{0}(\lambda)=\lambda^{-1 / 2}$ and so $\nu_{0}(\lambda)=1 / 2$. For an incompressible (area-preserving) elastic slab in 2D (i.e. when there is no direction $Z$ ), $\mu_{0}(\lambda)=\lambda^{-1}$ and $\nu_{0}(\lambda)=1$.

The second Piola-Kirchhoff stress $\underline{\underline{S}}_{0}(\lambda)=\underline{\underline{S}}\left(\underline{\underline{e}}_{0}(\lambda)\right)$ is uniaxial for the simple traction solution,

$$
\underline{\underline{S}}_{0}(\lambda)=\frac{n_{0}(\lambda)}{\lambda} \underline{e}_{X} \otimes \underline{e}_{X},
$$

where $n_{0}(\lambda)$ denotes the nominal stress, also called the engineering stress. The nominal stress $n_{0}(\lambda)$ is the derivative of the strain energy density $w_{0}(\lambda)=w\left(\underline{\underline{e}}_{0}(\lambda)\right)$ in simple traction ${ }^{1}$,

$$
n_{0}(\lambda)=\frac{\mathrm{d} w_{0}}{\mathrm{~d} \lambda}(\lambda)
$$

Consider now an equibiaxial solution $\underline{\underline{e}}_{0}(\lambda)$, plus an incremental shear deformation in a plane containing the material axis $X$. The response to this incremental shear deformation is described by linear orthotropic elasticity, since we consider an orthotropic hyperelastic material near an equibiaxial configuration whose main axis is aligned with that of the material symmetries. Let $G_{0}(\lambda)$ denote the corresponding tangent modulus, $G_{0}(\lambda)=A_{X Y X Y}\left(\underline{e}_{0}(\lambda)\right)=A_{X Z X Z}\left(\underline{\underline{e}}_{0}(\lambda)\right)=\cdots$ The orthotropy dictates the following identity:

$$
\underline{\underline{\underline{\underline{A}}}}\left(\underline{\underline{e}}_{0}(\lambda)\right):\left(\underline{e}_{X} \otimes \underline{e}_{J}+\underline{e}_{J} \otimes \underline{e}_{X}\right)=2 G_{0}(\lambda)\left(\underline{e}_{X} \otimes \underline{e}_{J}+\underline{e}_{J} \otimes \underline{e}_{X}\right), \quad \text { for } J=Y, Z
$$

with the same modulus $G_{0}(\lambda)$ for both kinds of shear deformations ( $J=Y$ or $Z$ ). This is the main property concerning material symmetry which we will use in the dimensional reduction.

\subsection{Coleman-Newman's construction}

We return to the prismatic bar and now consider non-uniform solutions. Let us denote by $\lambda(X)$ the prescribed macroscopic stretch, $\lambda(X)$. The deformed centerline is reconstructed by integration

$$
\Lambda(X)=\int_{0}^{X} \lambda\left(X^{\prime}\right) \mathrm{d} X^{\prime},
$$

up to a constant of integration representing a rigid-body translation. The actual distribution of the stretch $\lambda(X)$ will be found in the second part of the paper by solving the dimensionally reduced model. For the moment, the goal is to carry out this dimensional reduction, i.e. to calculate the energy in the bar in terms of $\lambda(X)$ in the limit $\epsilon \rightarrow 0$. Our dimensional reduction makes the key assumption that $\lambda(X)$ is a slowly varying function of $X$, i.e. a function which evolves on the long length-scale $L$ : mathematically, $\lambda(X)$ bears no dependence on $\epsilon$.

Assuming that the cross-sections remain planar and perpendicular to the centerline, Coleman and Newman (1988) have proposed an explicit transformation of the bar of the form:

$$
\underline{x}_{\mathrm{C}}(\underline{X})=\Lambda(X) \underline{e}_{x}+\mu_{0}(\lambda(X))\left(Y \underline{e}_{y}+Z \underline{e}_{z}\right) .
$$

A similar kinematical assumption has been used as a starting point by Antman and Carbone (1977). These constructions are based on the idea that each slice of the bar undergoes a rigid-body translation, plus the homogeneous equibiaxial transformation associated with the local value $\lambda(X)$ of the macroscopic stretch. We shall show that this type of kinematical assumption does not predict the asymptotically correct secondgradient bar model, even in the special case of a circular-cylindrical bar for which they were originally proposed. More precisely, they yield a one-dimensional model of the correct (second-gradient) type, but with

\footnotetext{
${ }^{1}$ Indeed, linearizing the generic expansion (2.3) about $\underline{\underline{e}}=\underline{\underline{e}}_{0}(\lambda)$ with an increment $\underline{\underline{\tilde{e}}}=\frac{\mathrm{d} \underline{\underline{e}}_{0}}{\mathrm{~d} \lambda}(\lambda) \tilde{\lambda}=(\lambda \underline{\underline{1}}) \tilde{\lambda}$ yields $w(\lambda+\tilde{\lambda})=$ $w(\lambda)+\underline{\underline{S}}_{0}(\lambda):(\lambda \underline{\underline{1}}) \tilde{\lambda}+\mathcal{O}\left(\tilde{\lambda}^{2}\right)$. Identifying this with the linear expansion of $w$, we have $\operatorname{tr} \underline{\underline{S}}_{0}(\lambda)=\frac{1}{\lambda} \frac{\mathrm{d} \bar{w}}{\mathrm{~d} \lambda}$, which is consistent with equations $(2 . \overline{7})$ and $(2.8)$.
} 
incorrect coefficients. The reason is that the ad hoc assumption of planar cross-sections in equation (2.11) is incompatible with the equilibrium of the lateral boundary, as we show later.

For later reference, the deformation gradient associated with Coleman-Newman's construction (2.11) reads

$$
\underline{\underline{F}}_{\mathrm{C}}=\left(\begin{array}{ccc}
\lambda & 0 & 0 \\
t Y & \mu & 0 \\
t Z & 0 & \mu
\end{array}\right)=\left(\begin{array}{ccc}
\lambda & 0 & 0 \\
\epsilon t \bar{Y} & \mu & 0 \\
\epsilon t \bar{Z} & 0 & \mu
\end{array}\right)
$$

where $\lambda=\lambda(X), \mu=\mu_{0}(\lambda(X)),(\bar{Y}, \bar{Z})$ are the stretched coordinates defined at the beginning of $\S 2.1$, and

$$
t(X)=\frac{\mathrm{d} \mu_{0}(\lambda(X))}{\mathrm{d} X}=\lambda^{\prime}(X) \mu_{0}^{\prime}(\lambda(X))=-\frac{\lambda^{\prime}(X) \mu_{0}(\lambda(X)) \nu_{0}(\lambda(X))}{\lambda(X)} .
$$

The third equality in the equation above follows from the definition of Poisson's ratio in equation (2.6). The quantity $t$ is proportional to the second gradient of displacement $\lambda^{\prime}$, and is a measure of the small conical angle of the lateral surface.

Our matrix notation for the tensor $\underline{\underline{F}}_{\mathrm{C}}$ in equation (2.12) makes reference to the Cartesian basis $\left(\underline{e}_{x}, \underline{e}_{y}, \underline{e}_{z}\right)$ : in tensor notation, $\underline{\underline{F}}_{\mathrm{C}}=\lambda \underline{\underline{e}}_{x} \otimes \underline{e}_{x}+\epsilon t \bar{Y} \underline{e}_{y} \otimes \underline{e}_{x}+\cdots$ Comparing with equation (2.4), it appears that this deformation gradient is identical to that of a homogeneous (equibiaxial) solution with stretch $\lambda(X)$, except for the small shear strain $\epsilon t\left(\bar{Y} \underline{e}_{y}+\bar{Z} \underline{e}_{z}\right) \otimes \underline{e}_{x}$. As we shall see, this residual shear strain gives rise to unbalanced shear stress, and we need to modifying Coleman-Newman's construction in order to relax it.

\subsection{Improving on Coleman-Newman's construction}

We seek an equilibrium solution for a slender bar in the form of a systematic expansion with respect to the aspect-ratio $\epsilon$,

$$
\underline{x}(\underline{X})=\left(\begin{array}{c}
\Lambda(X) \\
0 \\
0
\end{array}\right)+\epsilon\left[\mu_{0}(\lambda(X))\left(\begin{array}{c}
0 \\
\bar{Y} \\
\bar{Z}
\end{array}\right)+\left(\begin{array}{c}
0 \\
u_{y}(X, \bar{Y}, \bar{Z}) \\
u_{z}(X, \bar{Y}, \bar{Z})
\end{array}\right)\right]+\epsilon^{2}\left(\begin{array}{c}
u_{x}(X, \bar{Y}, \bar{Z}) \\
0 \\
0
\end{array}\right)+\epsilon^{3}\left(\begin{array}{c}
0 \\
\ldots \\
\ldots
\end{array}\right)+\cdots
$$

Note that the first two terms in the expansion correspond to Coleman-Newman's construction $\underline{x}_{\mathrm{C}}$ in $(2.11)$. Additional displacements $u_{x}, u_{y}, u_{z}$ have been introduced, which are functions of the stretched variables. The displacement is assumed to be axial when multiplied with even powers of $\epsilon$, and transverse when multiplied with odd powers, as required by the fact that the system is mirror-symmetric, $z \rightarrow-z$. The dots denote higher-order terms in the expansion, which are not required to capture the dominant contributions in the second gradient $\lambda^{\prime}(X)$.

The incremental displacement $\left(u_{x}, u_{y}, u_{z}\right)$ will now be adjusted to minimize the elastic energy in an asymptotic sense, i.e. for small $\epsilon$. To do so, we start by assigning an energy to any configuration $\Lambda(X)$, by minimizing the microscopic strain energy with a prescribed $\Lambda(X)$. We have defined $\Lambda(X)$ as the $x$-coordinate of the centroid of the deformed cross-section, see equation (2.1). In (2.14), we therefore require that the average of $u_{x}$ is zero on any cross-section: for any $X$,

$$
\iint_{\bar{\Omega}} u_{x}(X, \bar{Y}, \bar{Z}) \mathrm{d} \bar{Y} \mathrm{~d} \bar{Z}=0
$$

We start by calculating the deformation gradient $\underline{\underline{F}}$ based on the series expansion (2.14),

$$
\underline{\underline{F}}=\underline{\underline{F}}_{[0]}+\epsilon \underline{\underline{F}}_{[1]}+\epsilon^{2} \underline{\underline{F}}_{[2]}+\cdots
$$

At dominant order, we obtain

$$
\underline{\underline{F}}_{[0]}=F_{0}(\lambda(X))+\left(\begin{array}{ccc}
0 & 0 & 0 \\
0 & u_{y, \bar{Y}} & u_{y, \bar{Z}} \\
0 & u_{z, \bar{Y}} & u_{z, \bar{Z}}
\end{array}\right),
$$


where $\underline{\underline{F}}_{0}(\lambda)$ is the equibiaxial deformation gradient introduced in the analysis of simple traction, see equation (2.4). Note that the transverse gradients $\partial / \partial Y=\epsilon^{-1} \partial / \partial \bar{Y}$ and $\partial / \partial Z=\epsilon^{-1} \partial / \partial \bar{Z}$ shift the expansion orders by one unit, implying that subdominant (first-order) terms in the displacement end up as dominant terms in the deformation gradient $\underline{\underline{\underline{F}}}[0]$.

We require that the perturbation is small in the sense that the deformation gradient $\underline{\underline{F} \text { matches that of }}$ simple traction at dominant order $\epsilon=0$, i.e. $\underline{\underline{F}}_{[0]}=\underline{\underline{F}}_{0}(\lambda(X))$. In the equation above, this implies that $\left(u_{y}, u_{z}\right)$ are independent of $\bar{Y}$ and $\bar{Z}$, which we write as

$$
u_{y}(X, \bar{Y}, \bar{Z})=u_{y}(X), \quad u_{z}(X, \bar{Y}, \bar{Z})=u_{z}(X) .
$$

These $\left(u_{y}(X), u_{z}(X)\right)$ describe an infinitesimal rigid-body translation of cross-sections in their own planes.

At the following orders, the deformation gradient reads

$$
\underline{\underline{F}}_{[1]}=\left(\begin{array}{ccc}
0 & q_{Y} & q_{Z} \\
p_{y} & 0 & 0 \\
p_{z} & 0 & 0
\end{array}\right), \quad \underline{\underline{F}}_{[2]}=\left(\begin{array}{ccc}
u_{x}^{\prime}(X) & 0 & 0 \\
0 & \cdots & \cdots \\
0 & \cdots & \cdots
\end{array}\right),
$$

where the dots denote terms which we can ignore (and which depend on higher-order terms in the displacement), and

$$
\begin{array}{ll}
p_{y}=t(X) \bar{Y}+u_{y}^{\prime}(X) & q_{Y}=u_{x, \bar{Y}}(X, \bar{Y}, \bar{Z}) \\
p_{z}=t(X) \bar{Z}+u_{z}^{\prime}(X) & q_{Z}=u_{x, \bar{Z}}(X, \bar{Y}, \bar{Z}) .
\end{array}
$$

Commas in subscripts denote partial derivatives, as in $u_{x, \bar{Y}}=\frac{\partial u_{x}}{\partial \bar{Y}}$. Primes on the functions $u_{y}(X)$ and $u_{z}(X)$ unambiguously refer to derivation with respect to $X$. Note that $\underline{\underline{F}}_{\mathrm{C}}$ in $(2.12)$ can be recovered as a special case of $\underline{\underline{F}}_{[1]}$ in $(2.19)$ when $t=0$ in $(2.20)$.

From the expansion of $\underline{\underline{F}}$ just found, one can expand the Green-St-Venant tensor $\underline{\underline{e}}=\left(\underline{\underline{F}}^{T} \cdot \underline{\underline{F}}-\underline{\underline{1}}\right) / 2$ as

$$
\underline{\underline{e}}=\underline{\underline{e}}_{[0]}+\epsilon \underline{\underline{e}}_{[1]}+\epsilon^{2} \underline{\underline{e}}_{[2]}+\cdots
$$

where $\underline{\underline{e}}_{[0]}=\underline{\underline{e}}_{0}(\lambda(X))$ is the equibiaxial deformation gradient corresponding to simple traction, see (2.4),

$$
\underline{e}_{[1]}=\left(\begin{array}{ccc}
0 & \left(\mu p_{y}+\lambda q_{Y}\right) & \left(\mu p_{z}+\lambda q_{Z}\right) \\
\left(\mu p_{y}+\lambda q_{Y}\right) & 0 & 0 \\
\left(\mu p_{z}+\lambda q_{Z}\right) & 0 & 0
\end{array}\right), \quad \underline{e}_{[2]}=\left(\begin{array}{ccc}
e_{[2]}^{X X} & 0 & 0 \\
0 & \ldots & \ldots \\
0 & \ldots & \ldots
\end{array}\right)
$$

and the second-order correction to the axial strain reads

$$
e_{[2]}^{X X}=\frac{p_{y}^{2}+p_{z}^{2}}{2}+\lambda u_{x, X}(X, \bar{Y}, \bar{Z}) .
$$

In these equations, we use $\lambda$ and $\mu$ as a shorthand notation for the macroscopic stretch $\lambda(X)$ and the corresponding transverse stretch $\mu_{0}(\lambda(X))$ according to the simple traction solution, respectively.

The strain is therefore the sum of the equibiaxial one $\underline{\underline{e}}_{0}$ corresponding to simple traction (at dominant order $\epsilon^{0}=1$ ), plus a small shear strain which is itself the sum of Coleman-Newman's residual shear strain (terms proportional to $t$, hence to $\lambda^{\prime}(X)$, in $p_{y}$ and $p_{z}$ ) and of new terms that depend on the yet unknown incremental displacement $\left(u_{x}, u_{y}, u_{z}\right)$. In the forthcoming sections, we calculate the strain energy, and then adjust the displacement so as to minimize it.

\subsection{Expansion of the strain energy}

We now insert the special form of the strain found in equation (2.21a) into the expansion of the strain energy derived in equation (2.3): setting the base solution to be the simple traction solution $\underline{\underline{e}}_{0}=\underline{\underline{e}}_{0}(\lambda(X))$ and the increment to be $\underline{\underline{e}}_{\mathrm{C}}=\epsilon \underline{\underline{e}}_{[1]}+\epsilon^{2} \underline{\underline{e}}_{[2]}$, equation (2.3) yields

$$
w(\underline{\underline{e}})=w_{0}(\lambda(X))+\epsilon\left(\underline{\underline{S}}_{0}(\lambda(X)): \underline{\underline{e}}_{[1]}\right)+\epsilon^{2}\left(\underline{\underline{S}}_{0}(\lambda(X)): \underline{\underline{e}}_{[2]}+\frac{1}{2} \underline{\underline{e}}_{[1]}: \underline{\underline{\underline{\underline{A}}}}\left(\underline{\underline{e}}_{0}(\lambda(X))\right): \underline{\underline{e}}_{[1]}\right)+\cdots
$$


where $w_{0}(\lambda)=w\left(\underline{\underline{e}}_{0}(\lambda)\right), \underline{\underline{S}}_{0}(\lambda)$, and $\underline{\underline{\underline{\underline{A}}}}\left(\underline{\underline{e}}_{0}(\lambda)\right)$ denote the energy, the stress, and the tangent elasticity tensor about the simple traction solution, according to the notation introduced in $\S 2.3$.

The expansion for the strain energy can be simplified using the orthotropy property of the elasticity tensor in equation (2.9) and using the fact that $\underline{\underline{S}}_{0}(\lambda)=\frac{n_{0}(\lambda)}{\lambda} \underline{e}_{X} \otimes \underline{e}_{X}$ is uniaxial. Inserting the expansion of the strain found in equations $(2.21 \mathrm{~b}-2.21 \mathrm{c})$, we find that the first-order contribution to the energy cancels; to second-order, the expansion reads

$$
w(\underline{\underline{e}})=w_{0}(\lambda)+\epsilon^{2}\left(n_{0}(\lambda) u_{x, X}+\frac{n_{0}(\lambda)}{2 \lambda}\left(p_{y}^{2}+p_{z}^{2}\right)+2 G_{0}(\lambda)\left(\left(\mu p_{y}+\lambda q_{Y}\right)^{2}+\left(\mu p_{z}+\lambda q_{Z}\right)^{2}\right)\right)+\cdots
$$

where the $p$ 's and the $q$ 's are defined in equation $(2.20)$ and $G_{0}$ is the tangent shear modulus which has been introduced in equation (2.9). A similar expansion of the energy can be found in the 2D dimensional reduction of Mielke (1991).

Next, we integrate over the cross-section $\Omega$ and over the length $-L \leq X \leq L$, and calculate the strain energy $W=\int_{0}^{L} \iint_{\Omega} w(\underline{\underline{e}}) \mathrm{d} X \mathrm{~d} Y \mathrm{~d} Z$ as

$$
\begin{aligned}
& W=\int_{0}^{L} \iint_{\bar{\Omega}}\left[w_{0}(\lambda(X))+\epsilon^{2}\right.\left(\frac{n_{0}(\lambda(X))}{2 \lambda(X)}\left(t^{2}(X)\left(\bar{Y}^{2}+\bar{Z}^{2}\right)+u_{y}^{\prime 2}(X)+u_{z}^{\prime 2}(X)\right)\right. \\
&\left.\left.\cdots+2 G_{0}(\lambda(X))\left(\left(\mu p_{y}+\lambda q_{Y}\right)^{2}+\left(\mu p_{z}+\lambda q_{Z}\right)^{2}\right)\right)\right] \mathrm{d} X \mathrm{~d} \bar{Y} \mathrm{~d} \bar{Z} \epsilon^{2} .
\end{aligned}
$$

To simplify the right-hand side, we have used the fact that $u_{x}(X, \bar{Y}, \bar{Z})$ is zero on average on any crosssection, see (2.15), and as a result the first term in the parenthesis of (2.23) disappears upon integration, $\iint u_{x, X} \mathrm{~d} \bar{Y} \mathrm{~d} \bar{Z}=\frac{\mathrm{d}}{\mathrm{d} X} \iint u_{x} \mathrm{~d} \bar{Y} \mathrm{~d} \bar{Z}=0$; we have also expanded $p_{y}^{2}=\left(t \bar{Y}+u_{y}^{\prime}(X)\right)^{2}$ and observed that the cross-term $2 t(X) \bar{Y} u_{y}^{\prime}(X)$ disappears upon integration over the cross-section, since the origin $(\bar{Y}, \bar{Z})=(0,0)$ of the coordinate system has been chosen to coincide with the centroid of the cross-section, $\iint_{\bar{\Omega}} \bar{Y} \mathrm{~d} \bar{Y} \mathrm{~d} \bar{Z}=0$. The other term $p_{z}^{2}$ has been expanded similarly.

The energy $W$ in (2.24) must now be minimized with respect to the displacements $u_{x}(X, \bar{Y}, \bar{Z}), u_{y}(X)$ and $u_{z}(X)$, subjected to the constraint (2.15) of zero mean axial displacement $u_{x}$. The $p$ 's and the $q$ 's defined in (2.20) all depend linearly on the linear strains $\left(u_{x, \bar{Y}}, u_{x, \bar{Z}}, u_{y}^{\prime}, u_{z}^{\prime}\right)$, implying that $W$ is a quadratic function of the strains: the problem of finding the asymptotic displacement in a necked bar has been formulated as a problem of linear elasticity with residual strain. The residual strain arises from the terms proportional to $t$ (hence to the second gradient $\lambda^{\prime}$ ) in $p_{y}$ and $p_{z}$.

\subsection{Optimal displacement}

The problem of minimizing the strain energy $W$ in equation (2.24) with respect to the displacements has an obvious solution. Indeed, there exists a solution which cancels all the squared terms in the integrand that depend on the displacement,

$$
\begin{aligned}
u_{y}^{\prime}(X) & =0 \\
u_{z}^{\prime}(X) & =0 \\
\mu p_{y}+\lambda q_{Y} & =0 \\
\mu p_{z}+\lambda q_{Z} & =0 .
\end{aligned}
$$

The first two equations $(2.25 \mathrm{a}-2.25 \mathrm{~b})$ imply that $u_{y}$ and $u_{z}$ are constants. This corresponds to a transverse rigid-body displacement, which we can ignore:

$$
u_{y}(X)=0, \quad u_{z}(X)=0 .
$$

Inserting the definition (2.20) of the $p$ 's and the $q$ 's into the two other equations $(2.25 \mathrm{c}-2.25 \mathrm{~d})$, we find $u_{x, \bar{Y}}=-\frac{\mu}{\lambda} t(X) \bar{Y}$ and a similar equation $u_{x, \bar{Z}}=-\frac{\mu}{\lambda} t(X) \bar{Z}$. Remarkably, these equations are geometrically 
compatible, i.e. $\left(u_{x, \bar{Y}}\right)_{\bar{Z}}=0=\left(u_{x, \bar{Z}}\right)_{\bar{Y}}$ : an integration yields the axial displacement as

$$
u_{x}(X, \bar{Y}, \bar{Z})=-\frac{\mu_{0}(\lambda(X))}{2 \lambda(X)} t(X)\left(\left(\bar{Y}^{2}+\bar{Z}^{2}\right)-\left\langle\bar{Y}^{2}+\bar{Z}^{2}\right\rangle\right) .
$$

Here, $\left\langle\bar{Y}^{2}+\bar{Z}^{2}\right\rangle$ is a geometrical constant, defined as the cross-section average of $\bar{Y}^{2}+\bar{Z}^{2}$ : this constant of integration has been chosen such that the constraint (2.15) is satisfied, i.e. the average axial displacement is zero on any cross-section.

In equation above, the term $\left(\bar{Y}^{2}+\bar{Z}^{2}\right)$ captures the curvature of cross-sections. Also, note that the quantities $\mu p_{y}+\lambda q_{Y}$ and $\mu p_{z}+\lambda q_{Z}$ which we have canceled in equations $(2.25 \mathrm{c}-2.25 \mathrm{~d})$ are the incremental shear strain $e_{[1]}^{x y}$ and $e_{[1]}^{x z}$ : they first appeared in the linearized strain $\underline{\underline{e}}_{[1]}$ in equation $(2.21 \mathrm{~b})$. The curvature of the cross-sections warrants the cancellation of the linearized shear strain,

$$
e_{[1]}^{x y}(X, \bar{Y}, \bar{Z})=0, \quad e_{[1]}^{x z}(X, \bar{Y}, \bar{Z})=0 .
$$

This shows that the deformed cross-sections remain locally perpendicular to material lines initially parallel to the axis. For non-homogeneous stretch $\lambda(X)$, these material lines are convergent or divergent, and as a result the cross-sections will be curved, see figure $1 \mathrm{c}$.

\subsection{Summary: energy of the second-gradient model}

Inserting the optimal solution (2.25) into the expansion (2.24) of the elastic energy, we find that the optimal energy of the bar is given asymptotically for $\epsilon \rightarrow 0$ by a one-dimensional model,

$$
W=\int_{-L}^{L}\left[A w_{0}(\lambda(X))+\frac{B(\lambda(X))}{2}\left(\frac{\mathrm{d} \lambda}{\mathrm{d} X}\right)^{2}\right] \mathrm{d} X,
$$

where $A=\iint_{\Omega} \mathrm{d} Y \mathrm{~d} Z$ is the undeformed cross-sectional area. After using the definition of $t(X)$ in equation (2.13), the modulus associated with the second-gradient terms is found as

$$
B(\lambda)=n_{0}(\lambda) \frac{\nu_{0}^{2}(\lambda) \mu_{0}^{2}(\lambda)}{\lambda^{3}} I \quad(\text { general case })
$$

where $I$ is the geometric moment of inertia of the cross-section,

$$
I=\iint_{\Omega}\left(Y^{2}+Z^{2}\right) \mathrm{d} Y \mathrm{~d} Z
$$

In the integrand in equation (2.28a), the first term is the classical energy of a bar depending on the first gradient of the displacement $\lambda=\Lambda^{\prime}(X)$ (stretch), and the second term is a second-gradient term depending on $\lambda^{\prime}=\Lambda^{\prime \prime}(X)$ (gradient of stretch). Here, recall that $\Lambda(X)=\langle x\rangle(X)$ denotes the position of the centroid of a deformed cross-section, see (2.1).

\subsection{Special cases}

Our derivation of the second-gradient modulus $B$ in equation $(2.28 \mathrm{~b})$ holds in general for a threedimensional prismatic solid having an arbitrary cross-section, made of an orthotropic material. The dependence on the geometry of the cross-section is entirely captured by the geometric moment of inertia I. $\lambda^{-1 / 2}$ :

The special case of a three-dimensional incompressible material is found by setting $\nu_{0}=1 / 2$ and $\mu_{0}(\lambda)=$

$$
B=\frac{n_{0}(\lambda) I}{4 \lambda^{4}} \quad \text { (incompressible 3D prismatic solid). }
$$


For a circular-cylindrical bar having diameter $D, I=\frac{\pi D^{4}}{32}$, and so

$$
B=\frac{\pi D^{4}}{128 \lambda^{4}} n_{0}(\lambda) \quad \text { (incompressible circular cylinder) }
$$

In the entire derivation of the second-gradient model, the transverse directions $Y$ and $Z$ are uncoupled. As a result, the derivation can easily be specialized to the case of a long 2D slab in plane strain by dropping all quantities bearing an index $z$ or $Z$. Let $h \times(2 L)$ be the undeformed dimensions of the slab, $\Omega=[-h / 2, h / 2]$, with $h \ll 2 L$. The result is simply $B=n_{0}(\lambda) \frac{\nu_{0}^{2}(\lambda) \mu_{0}^{2}(\lambda)}{\lambda^{3}} I_{2 D}$, where $I_{2 D}=\int_{-h / 2}^{h / 2} Y^{2} \mathrm{~d} Y=\frac{h^{3}}{12}$ :

$$
\left.B=\frac{h^{3}}{12} \frac{\nu_{0}^{2}(\lambda) \mu_{0}^{2}(\lambda)}{\lambda^{3}} n_{0}(\lambda) \quad \text { (compressible } 2 \mathrm{D} \text { block }\right)
$$

In the $2 \mathrm{D}$ incompressible (area-preserving) case, we have $\nu_{0}=1, \mu_{0}(\lambda)=\lambda^{-1}$ and so

$$
B=\frac{h^{3}}{12 \lambda^{5}} n_{0}(\lambda) \quad \text { (incompressible } 2 \mathrm{D} \text { block). }
$$

The energy of the second gradient model announced in equations (1.2) and (1.4) for an incompressible 2D slab and for an incompressible 3D circular cylindrical bar follow from these expressions. In those expressions, we introduced a dimensionless modulus $b$ of the second-gradient term by dividing the physical modulus $B$ by the diameter/area of the cross-section $\Omega$ times a suitable power of the diameter, $b=\frac{B}{h \times h^{2}}$ in $2 \mathrm{D}$, and $b=\frac{B}{\frac{\pi D^{2}}{4} \times D^{2}}$ in $3 \mathrm{D}$.

\subsection{Microscopic displacement}

In view of the expansion (2.14) and of the solution found in $\S 2.7$, the optimal solution reads

$$
\underline{x}(\underline{X})=\Lambda(X) \underline{e}_{x}+\mu_{0}(\lambda(X))\left(Y \underline{e}_{Y}+Z \underline{e}_{Z}\right)+\lambda^{\prime}(X) c(\lambda(X))\left(\frac{Y^{2}+Z^{2}}{2}-\frac{I}{2 A}\right) \underline{e}_{x}+\cdots
$$

where the dots denote higher-order terms in $\epsilon$. The constant term warranting the cancellation of the average axial displacement has been rewritten as $\left\langle Y^{2}+Z^{2}\right\rangle=\iint\left(Y^{2}+Z^{2}\right) \mathrm{d} Y \mathrm{~d} Z / A=I / A$, where $I$ is the geometric moment of inertia and $A$ the area.

In the right-hand side of equation (2.30a), the first term represents the displacement $\Lambda(X)$ averaged on a cross-section, the second term is the transverse contraction of the cross-section by Poisson's effect (as captured by Coleman-Newman), and the third term describes the curvature of the cross-section. The curvature coefficient $c(\lambda)$ is such that there is no shear strain at dominant order, and reads

$$
c(\lambda)=\frac{\mu_{0}^{2}(\lambda) \nu_{0}(\lambda)}{\lambda^{2}}
$$

Even though the curvature enters into the displacement at order $\epsilon^{2}$, and may be thought to be negligible compared to the linear term included in Coleman-Newman's construction, it contributes to the deformation gradient - and hence to the strain energy - at the same order $\epsilon$, see (2.19).

In the special case of an incompressible material, these expressions yield those announced in equation (1.1) for a $2 \mathrm{D}$ slab, and in equation (1.3) for a circular cylindrical bar in 3D.

\subsection{Discussion}

Equations (2.28a-2.28b) define the one-dimensional model for necking, and are the main result of this section. It is remarkable that the modulus $B$ of the second-gradient term in $(2.28 \mathrm{~b})$ can be expressed directly in terms of the functions $n_{0}(\lambda), \nu_{0}(\lambda)$ and $\mu_{0}(\lambda)$ which characterize the non-linear response to simple traction, see $\S 2.3$. We have carried out the dimensional reduction for a generic material with orthotropy aligned with the undeformed axis - but there is no shear in the solution to first order in $\lambda^{\prime}$, the modulus $B(\lambda)$ ultimately 
does not depend on the shear modulus $G_{0}$. To the best of our knowledge, the simple formula (2.28b) for the second-gradient term is novel; also, in previous work, the dimensional reduction has been carried out rigorously in the two-dimensional case only, by Mielke (1991). In addition, an upper bound for the second-gradient modulus $B$ has been derived by Coleman and Newman (1988) in the particular case of an axisymmetric geometry, see the discussion in $\$ 2.11$.

To first order in $\lambda^{\prime}$, the kinematics of the solution gives rise to zero shear straining parallel to the free surface, see equation (2.27) and figure 1. Using the constitutive law, this implies in particular that the shear stress tangent to the free surface is zero as required by the equilibrium, see $\S 2.12$.

Coleman-Newman's assumption of planar cross-sections corresponds to canceling the axial displacement, $u_{x}^{\mathrm{C}}(X, \bar{Y}, \bar{Z})=0$, in the expansion (2.14). The corresponding values of the deformation gradients are $p_{y}^{\mathrm{C}}=$ $t(X) \bar{Y}, p_{z}^{\mathrm{C}}=t(X) \bar{Z}, q_{Y}^{\mathrm{C}}=0$ and $q_{Z}^{\mathrm{C}}=0$. The associated first-order shear straining is $e_{[1] \mathrm{C}}^{x y}=\mu p_{y}^{\mathrm{C}}=\mu t \bar{Y}$ and a similar formula for $e_{[1] \mathrm{C}}^{x y}$. In the strain energy, this gives rise to an additional shear contribution, coming from the second line in equation (2.24),

$$
W_{\mathrm{C}}=W+\epsilon^{2} \int_{0}^{L} \iint_{\bar{\Omega}} 2 G_{0} \mu_{0}^{2}(\lambda) t^{2}(X)\left(\bar{Y}^{2}+\bar{Z}^{2}\right) \mathrm{d} \bar{Y} \mathrm{~d} \bar{Z} \epsilon^{2}+\mathcal{O}\left(\epsilon^{6}\right) .
$$

This term is proportional to $\lambda^{\prime 2}$ since $t$ is proportional to $\lambda^{\prime}$. This means that the energy $W_{\mathrm{C}}$ is of the same (second-gradient) type as the correct 1D energy $W$, but with a second-gradient modulus strictly larger than the exact one. The ad hoc kinematical assumption of planar cross-sections therefore yields an upper bound for the elastic energy, as could be anticipated.

For an isotropic material, for example, the spurious term in the strain energy (2.31) above gives rise to a prediction for the second-gradient modulus $B_{\mathrm{C}}(\lambda)=n_{0}(\lambda) \frac{\nu_{0}^{2}(\lambda) \mu_{0}^{2}(\lambda)}{\lambda^{3}} \frac{\lambda^{2}}{\lambda^{2}-\mu_{0}^{2}(\lambda)} I$, to be contrasted with the exact formula in (2.28b). For the particular case of a $2 \mathrm{D}$ block in plane strain made of an incompressible material $\left(\mu_{0}=\lambda^{-1}\right)$, this yields, after rescaling,

$$
b_{\mathrm{C}}(\lambda)=\frac{n_{0}(\lambda)}{12 \lambda^{5}} \frac{\lambda^{4}}{\lambda^{4}-1} \quad \text { (upper bound, incompressible 2D block). }
$$

For the particular case of a circular cylinder made of an incompressible material $\left(\mu_{0}=\lambda^{-1 / 2}\right)$, this yields, after rescaling,

$$
b_{\mathrm{C}}(\lambda)=\frac{n_{0}(\lambda)}{32 \lambda^{4}} \frac{\lambda^{3}}{\lambda^{3}-1} \quad \text { (upper bound, axisym. cylinder). }
$$

These are indeed the formulae proposed ${ }^{2}$ by Coleman and Newman (1988). Noting that both $\frac{\lambda^{4}}{\lambda^{4}-1}$ and $\frac{\lambda^{3}}{\lambda^{3}-1}$ are always larger than 1 because of the assumption $\lambda>1$ made in equation (2.5), and contrasting with the exact formulae $b=\frac{n_{0}(\lambda)}{12 \lambda^{5}}$ in (1.2) and $b=\frac{n_{0}(\lambda)}{32 \lambda^{4}}$ in (1.4), we can confirm that the modulus is always overestimated $^{3}, b_{\mathrm{C}}>b$ : it is based on a kinematical Ansatz which induces shear straining to the lowest order in $\lambda^{\prime}$, and does not satisfy the stress-free condition on the lateral boundary.

The error in the moduli $b_{\mathrm{C}}$ predicted by $(2.32 \mathrm{a})$ or $(2.32 \mathrm{~b})$ is not small: typically, for the point ' $\mathrm{B}$ ' shown later in figure 3 corresponding to a relative early stage of necking, $\lambda \approx 1.3$, so $\frac{\lambda^{4}}{\lambda^{4}-1}=1.54$ and $\frac{\lambda^{3}}{\lambda^{3}-1}=1.81$, implying that Coleman-Newman's construction overestimates the second-gradient modulus $b$ by as much as $50 \%$ for a $2 \mathrm{D}$ block in plane strain, and $80 \%$ for a circular cylinder.

\footnotetext{
${ }^{2}$ Equations [56] and [44] of Coleman and Newman (1988) can be simplified and are equivalent to our equations (2.32a) and $(2.32 \mathrm{~b})$, respectively. Take the $2 \mathrm{D}$ case, for instance: from equation (2.8), the engineering stress in simple traction is $n_{0}(\lambda)=D W \frac{\mathrm{d} \hat{\psi}\left(\lambda^{2}, \lambda^{-2}, 1\right)}{\mathrm{d} \lambda}$ in their own notation. Expanding the total derivative in the right-hand side, we have $n_{0}(\lambda)=$ $2 \lambda D W\left[\partial_{1} \hat{\psi}\left(\lambda^{2}, \lambda^{-2}, 1\right)-\lambda^{-4} \hat{\psi}\left(\lambda^{2}, \lambda^{-2}, 1\right)\right]$. Their equation [56] can then be simplified by identifying the expression appearing in their square bracket with that just obtained, namely $n_{0}(\lambda) /(2 \lambda D W)$. This yields $\frac{(-\bar{\gamma})}{D / W}=\frac{n_{0}(\lambda)}{12 \lambda^{5}} \frac{\lambda^{4}}{\lambda^{4}-1}$, as announced in equation (2.32a) above. The equivalence of the formulae for the axisymmetric case follows by a similar argument.

${ }^{3}$ The same conclusion can actually be reached in the compressive case $(\lambda<1)$, which we do not study here.
} 


\subsection{Microscopic stress}

To reconstruct the microscopic stress associated with our solution, we expand the second Piola-Kirchhoff stress near the simple traction solution $\underline{\underline{e}}_{0}=\underline{\underline{e}}_{0}(\lambda)$ as $\underline{\underline{S}}\left(\underline{\underline{e}}_{0}+\epsilon \underline{\underline{e}}_{[1]}\right)=\underline{\underline{S}}\left(\underline{\underline{e}}_{0}\right)+\epsilon \underline{\underline{\underline{A}}}\left(\underline{\underline{e}}_{0}(\lambda)\right): \underline{\underline{e}}_{[1]}$, where $\underline{\underline{\underline{\underline{A}}}}$ is the orthotropic elasticity tensor introduced in (2.9). The optimal solution derived in $\S 2.7$ has no shear, $\underline{\underline{\underline{e}}}_{[1]}=\underline{\underline{0}}$. As a result, the stress stays unperturbed to first order,

$$
\underline{\underline{S}}(X, Y, Z)=\frac{n_{0}(\lambda(X))}{\lambda(X)} \underline{e}_{X} \otimes \underline{e}_{X}+\underline{\underline{0}} \epsilon+\mathcal{O}\left(\epsilon^{2}\right) .
$$

In particular, $\underline{S}$ does not depend on the gradient of stretch $\lambda^{\prime}(X)$ and is constant on any cross-section, to first order in $\lambda^{\bar{T}}$. The stress measure in equation (2.33) is purely Lagrangian, and stays uniaxial with a fixed direction. This means that the Cauchy stress is locally uniaxial, but with a principal direction rotating along with the material: it stays aligned with the material direction initially parallel to the $X$-axis.

In particular, equation (2.33) warrants that the surface traction $\underline{\underline{S}} \cdot \underline{n}$ is always zero to order $\epsilon$, as required by the equilibrium of the lateral boundary. Here $\underline{n}$ is the unit normal in reference configuration, such that $\underline{n} \cdot \underline{e}_{X}=0$.

The next correction in (2.33), of order $\epsilon^{2}$, can be determined by solving the equations of equilibrium to second order, as Bridgman (1952) does for a circular cross-section: he finds a hydrostatic stress with a parabolic profile in the cross-section, which cancels on the boundary.

\section{Applications to nonlinear elastic materials}

In this section the accuracy of the one-dimensional model is demonstrated: we compare its prediction for the stretch at which the onset of necking occurs for a block under tension, with the results of an exact bifurcation analysis of the same problem. The one-dimensional model is then applied to predict the full necking response of a nonlinear elastic material that has a maximum load in uniaxial tension. Attention will focus on the block, but it is evident from the close similarity between the two energy functionals in equations (1.2) and (1.4), that the necking behavior of the round bar will be qualitatively similar.

In the rest of the paper, we assume that the material is incompressible, and consider necking solutions that are symmetric with respect to the center $X=0$ of the bar, see figure 1 . By symmetry, we consider the domain comprising the right-hand side half of the bar, $0 \leq X \leq L$. At the center of the neck at $X=0$, both the horizontal displacement and the shear traction vanish by symmetry. Idealized conditions are imposed on the right end-plane at $X=L$, such that the shear tractions vanish and the displacement is equated to $\delta$ (for the full bar, the end-to-end displacement is $2 \delta$, see figure 1 ).

\subsection{Bifurcation analysis of the onset of necking: comparison with exact results}

Consider the plane strain deformation of a rectangular block with thickness $h$ and length $2 L$ in the undeformed state. The uniform stretch in the pre-bifurcation state is $\lambda_{0}=1+\delta / L$. In accord with the one-dimensional model described in the Introduction, consider an incompressible isotropic nonlinear elastic solid with energy density in plane strain characterized by $w(\lambda)$ - this $w(\lambda)$ is the function called $w_{0}(\lambda)$ in $\S 2$, but we drop all subscripts ' 0 ' from now on for the sake of readability. It is readily established using equation (1.2) that the one-dimensional model admits only the uniform stretch solution if the energy density obeys $\frac{\mathrm{d}^{2} w(\lambda)}{\mathrm{d} \lambda^{2}}>0$, i.e. if the engineering stress for homogeneous solutions $n(\lambda)$ is such that $\frac{\mathrm{d} n(\lambda)}{\mathrm{d} \lambda}>0$. This is the case, for example, for a neo-Hookean material. Bifurcation from the uniform state can only occur if the energy density admits a maximum nominal stress in plane strain tension, i.e. a maximum of $n(\lambda)=\frac{\mathrm{d} w(\lambda)}{\mathrm{d} \lambda}$. Assume this is the case and denote by $\lambda_{M}$ the Considère stretch satisfying $\frac{\mathrm{d}^{2} w}{\mathrm{~d} \lambda^{2}}\left(\lambda_{M}\right)=0$.

Bifurcation from the uniform state is considered with $\lambda(X)=\lambda_{0}+\hat{\lambda}(X)$ with $\frac{\mathrm{d} \hat{\lambda}}{\mathrm{d} X}(X=0)=\frac{\mathrm{d} \hat{\lambda}}{\mathrm{d} X}(X=$ $L)=0$, consistent with symmetry at the center and the traction-free condition at the right end. The first bifurcation occurs in the mode $\hat{\lambda}=\cos \frac{\pi X}{L}$ at a critical stretch $\lambda_{0}=\lambda_{\text {c given by }}$

$$
-\frac{\mathrm{d}^{2} w}{\mathrm{~d} \lambda^{2}}\left(\lambda_{\mathrm{c}}\right)=\left(\frac{\pi h}{L}\right)^{2} \frac{1}{12 \lambda_{\mathrm{c}}^{5}} \frac{\mathrm{d} w}{\mathrm{~d} \lambda}\left(\lambda_{\mathrm{c}}\right) .
$$


In the right-hand side, the dimensionless modulus of the second-gradient term (called $b\left(\lambda_{\mathrm{c}}\right)$ earlier) has appeared: compare with equation (1.2). This equation for the bifurcation stretch $\lambda_{\mathrm{c}}$ is classically obtained by solving the linearized equations of equilibrium obtained from the second-gradient model (1.2) by the Euler-Lagrange method. An alternative derivation is presented later in $\S 3.2$, by taking the small-amplitude limit of an explicit nonlinear solution.

It is insightful to express (3.1) in terms of variables in the uniformly deformed state at the bifurcation stretch $\lambda_{c}$. To this end, in the uniform state, denote the $\operatorname{logarithmic}$ strain by $\varepsilon=\ln \lambda$, the true (Cauchy) stress by $\sigma=\lambda \frac{\mathrm{d} w(\lambda)}{\mathrm{d} \lambda}$, and the incremental (tangent) modulus of the true stress-logarithmic strain relation by $E_{\mathrm{t}}=\frac{\mathrm{d} \sigma}{\mathrm{d} \varepsilon}=\lambda \frac{\mathrm{d} \sigma}{\mathrm{d} \lambda}=\lambda^{2} \frac{\mathrm{d}^{2} w}{\mathrm{~d} \lambda^{2}}+\sigma$. Thus, $\frac{\mathrm{d}^{2} w}{\mathrm{~d} \lambda^{2}}\left(\lambda_{\mathrm{c}}\right)=\left(E_{\mathrm{t}}^{\mathrm{c}}-\sigma_{\mathrm{c}}\right) \lambda_{\mathrm{c}}^{-2}$ such that (3.1) becomes

$$
\sigma_{\mathrm{c}}-E_{\mathrm{t}}^{\mathrm{c}}=\left(\frac{\pi \bar{h}}{\bar{L}}\right)^{2} \frac{\sigma_{\mathrm{c}}}{12}
$$

with $\bar{h}=h / \lambda_{\mathrm{c}}$ and $\bar{L}=\lambda_{\mathrm{c}} L$ as the thickness and half-length of the block at bifurcation. By the same equation $E_{\mathrm{t}}=\lambda^{2} \frac{\mathrm{d}^{2} w}{\mathrm{~d} \lambda^{2}}+\sigma$, Considère's criterion of maximum nominal stress $\frac{\mathrm{d}^{2} w}{\mathrm{~d} \lambda^{2}}\left(\lambda_{\mathrm{m}}\right)=0$ is equivalent to the condition $\sigma_{\mathrm{m}}=E_{\mathrm{t}}^{\mathrm{m}}$, which is known as Considère's criterion for the true stress at the onset of necking. Equation (3.2) predicts that the onset of necking is delayed beyond Considère's criterion.

An exact analysis of the two-dimensional bifurcation problem for the block of the same material with the same boundary conditions has been given by Hill and Hutchinson (1975), and it also predicts that bifurcation is delayed beyond attainment of the maximum nominal stress. In the uniform deformed state at bifurcation the true stress at bifurcation from that analysis is given by their equation [6.7],

$$
\left.\frac{\sigma_{\mathrm{c}}}{E_{\mathrm{t}}^{\mathrm{c}}}\right|_{\mathrm{HH}}=1+\frac{1}{3} \gamma^{2}+\frac{7}{45} \gamma^{4}+O\left(\gamma^{6}, \frac{E_{\mathrm{t}}^{\mathrm{c}}}{G_{\mathrm{t}}^{\mathrm{c}}} \gamma^{6}\right) \quad(\text { exact, } 2 \mathrm{D}),
$$

with $\gamma=\frac{1}{2} \frac{\pi \bar{h}}{\bar{L}}$ and $G_{\mathrm{t}}^{\mathrm{c}}$ as the incremental shear modulus at bifurcation. Numerical results in Hill and Hutchinson (1975) reveal that equation (3.3) is accurate for $\gamma$ as large as $\gamma \simeq 1$ and only weakly dependent of $G_{\mathrm{t}}^{\mathrm{c}}$ and as long as $E_{\mathrm{t}}^{\mathrm{c}} / G_{\mathrm{t}}^{\mathrm{c}}<1$. The result from the one-dimensional model (3.2) can be expressed in the same manner as

$$
\frac{\sigma_{\mathrm{c}}}{E_{\mathrm{t}}^{\mathrm{c}}}=\left(1-\frac{\gamma^{2}}{3}\right)^{-1} \quad(\text { asymptotically exact, } 1 \mathrm{D}) .
$$

This can be expanded as $\frac{\sigma_{c}}{E_{t}^{\mathrm{c}}}=1+\frac{1}{3} \gamma^{2}+\frac{1}{9} \gamma^{4}+\cdots$ Comparison with equation (3.3) shows that the error in the one-dimensional model is of order $\gamma^{4}$, which is consistent with the order of approximation.

It is informative to compare with the prediction based on the approximate one-dimensional model of Coleman-Newman, which for plane strain has an additional factor $\lambda^{4} /\left(\lambda^{4}-1\right)$, see $\S 2.11$ :

$$
\left.\frac{\sigma_{\mathrm{c}}}{E_{\mathrm{t}}^{\mathrm{c}}}\right|_{\mathrm{C}}=\left(1-\frac{\lambda^{4}}{\lambda^{4}-1} \frac{\gamma^{2}}{3}\right)^{-1} \quad \text { (upper-bound, 1D). }
$$

The expansion $\left.\frac{\sigma_{\mathrm{c}}}{E_{\mathrm{t}}^{\mathrm{c}}}\right|_{\mathrm{C}}=1+\frac{1}{3} \frac{\lambda^{4}}{\lambda^{4}-1} \gamma^{2}+\cdots$ does not agree with (3.3) beyond the obvious constant term 1, and this confirms that this model is not asymptotically exact.

A number of numerical results will be presented later in the paper for a specific simple power-law material widely used to model aspects of plasticity in ductile metals. This incompressible, isotropic material model can also be used to illustrate the accuracy of the one-dimensional model in predicting the onset of necking. In plane strain, the power law material is defined by

$$
w(\lambda)=\frac{\sigma^{*}}{N+1}(\ln \lambda)^{N+1}=\frac{\sigma^{*}}{N+1} \varepsilon^{N+1}
$$

where $\lambda>1$ is the maximum principal stretch, $\varepsilon=\ln \lambda, N$ is called the strain hardening exponent $(0<$ $N<1$ ), and $\sigma^{*}$ is a reference stress. In plane strain tension, the true stress is $\sigma=\sigma^{*} \varepsilon^{N}$ with $\varepsilon=\ln \lambda$, the 
(a)

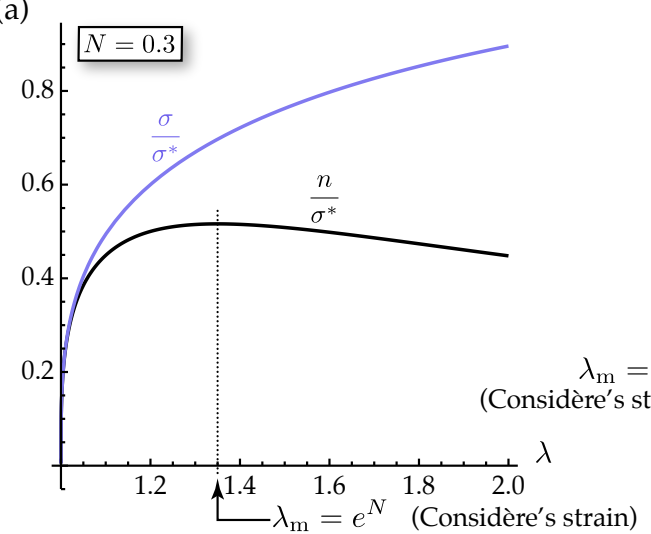

(b)

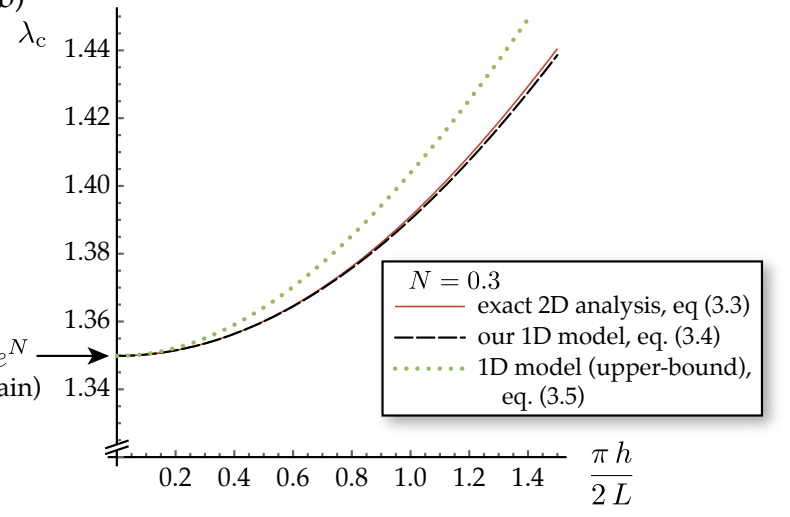

Figure 2: (a) The true stress $\sigma$ and the nominal stress $n$ for the power law material defined by equation (3.6) with $N=0.3$. (b) An example comparing the stretch at bifurcation for a block of thickness $h$ and half-length $L$ in the undeformed state, as derived from the exact two-dimensional analysis (3.3) by Hill and Hutchinson (1975), from the one-dimensional model (3.4) derived in this paper, and from the approximate one-dimensional model (3.5) of Coleman and Newman (1988).

nominal stress is $n=\sigma / \lambda=\sigma^{*}(\ln \lambda)^{N} / \lambda$, the Considère logarithmic strain at the maximum nominal stress is $\varepsilon_{\mathrm{m}}=N$, and the quantity appearing in the left-hand sides above is $\frac{\sigma}{E_{\mathrm{t}}}=(\ln \lambda) / N$. The true and nominal stress are plotted in figure $2 \mathrm{a}$ for $N=0.3$. The stretch at bifurcation, $\lambda_{\mathrm{c}}$, as obtained from the exact $2 \mathrm{D}$ theory (3.3), from the asymptotically exact one-dimensional model (3.4), and from the approximate onedimensional model (3.5), are plotted in figure $2 \mathrm{~b}$, still for $N=0.3$ : it can be seen that the one-dimensional model (3.4) is very accurate even for quite stocky blocks. By contrast, the one-dimensional model (3.5) based on an upper bound gives much less accurate results; consistent with the fact that it overestimates the energy of the bifurcated (necked) solution, this model delays the bifurcation artificially, i.e. provides an upper bound for the critical strain. For relatively slender blocks, i.e. $\frac{\pi h}{2 L}<0.5$, all three models predict a critical strain at the onset of necking which is very close to Considère estimate $\lambda_{\mathrm{m}} \approx 1.35$.

\subsection{Necking behavior of a block of nonlinear elastic material}

We proceed to investigate the post-bifurcation behavior of the same block considered above. For prescribed uniform nominal stress $n$ on the ends, the energy of the system is the sum of the $1 \mathrm{D}$ energy $W(\lambda)$ of the block and the potential energy $E_{\mathrm{p}}$,

$$
W(\lambda)+E_{\mathrm{p}}(\lambda)=h \int_{0}^{L}\left[w(\lambda)+\frac{h^{2}}{2} b(\lambda)\left(\frac{\mathrm{d} \lambda}{\mathrm{d} X}\right)^{2}-n \lambda\right] \mathrm{d} X,
$$

with $b(\lambda)$ defined in equation (1.2) and $n$ now independent of $X$. Stationarity of $W+E_{\mathrm{p}}$ with respect to variations of the stretch $\lambda(X)$ generates the second order ordinary differential equation and boundary conditions,

$$
\begin{array}{cc}
-h^{2} b(\lambda) \frac{\mathrm{d}^{2} \lambda}{\mathrm{d} X^{2}}-\frac{h^{2}}{2} \frac{\mathrm{d} b}{\mathrm{~d} \lambda}(\lambda) & \left(\frac{\mathrm{d} \lambda}{\mathrm{d} X}\right)^{2}+\frac{\mathrm{d} w}{\mathrm{~d} \lambda}(\lambda)=n \\
\frac{\mathrm{d} \lambda}{\mathrm{d} X}(0)=0 & \frac{\mathrm{d} \lambda}{\mathrm{d} X}(L)=0 .
\end{array}
$$

This non-linear boundary value problem can be integrated numerically in a straightforward manner using arc-length continuation (Doedel et al., 2007). Alternatively, it can also be solved by quadrature, thanks to the existence of a first integral,

$$
C=w(\lambda)-\frac{h^{2}}{2} b(\lambda)\left(\frac{\mathrm{d} \lambda}{\mathrm{d} X}\right)^{2}-n \lambda .
$$


This first integral is used to construct the solution below. In this respect, our approach has close parallels to the work of Coleman and Newman (1988) that developed and employed a one-dimensional model to study neck development and propagation relevant to polymer drawing processes. These authors considered nonlinear elastic materials whose nominal stress-strain curves in tension display the up-down-up behavior of some polymers that gives rise to the formation of a localized neck followed by its spread along the block or bar. The power-law material under consideration here is characteristic of metal in that the nominal stress in tension falls monotonically after the maximum load is attained. Consequently, once it forms, the neck localizes the deformation and does not spread. Assume the center of the neck is at $X=0$ with symmetry about the center. Denote the stretches at the center and at the right end by $\lambda_{L}=\lambda(X=0)$ and $\lambda_{R}=\lambda(X=L)$, with $\lambda_{L}>\lambda_{R}$ in the bifurcated solution. The conditions $\frac{\mathrm{d} \lambda}{\mathrm{d} X}=0$ at $X=0$ and $X=L$ imply $C=w\left(\lambda_{L}\right)-n \lambda_{L}=w\left(\lambda_{R}\right)-n \lambda_{R}$. As a result, we have

$$
n=\frac{w\left(\lambda_{L}\right)-w\left(\lambda_{R}\right)}{\lambda_{L}-\lambda_{R}},
$$

and the invariant $(3.9)$ can be rewritten as

$$
\sqrt{\frac{b(\lambda)}{2}} h \frac{\mathrm{d} \lambda}{\mathrm{d} X}=-\Delta
$$

where we have defined $\Delta=\left[\left(\lambda_{L}-\lambda\right) n-\left(w\left(\lambda_{L}\right)-w(\lambda)\right)\right]^{1 / 2}$, and we have assumed $\frac{\mathrm{d} \lambda}{\mathrm{d} X}<0$ on the interval $0<X<L$, in accord with $\lambda_{L}>\lambda_{R}$. Inserting the expression of $n$ in equation $(3.10), \Delta$ reads

$$
\Delta\left(\lambda_{L}, \lambda_{R}, \lambda\right)=\sqrt{\lambda_{L}-\lambda}\left[\frac{w\left(\lambda_{L}\right)-w\left(\lambda_{R}\right)}{\lambda_{L}-\lambda_{R}}-\frac{w\left(\lambda_{L}\right)-w(\lambda)}{\lambda_{L}-\lambda}\right]^{1 / 2} .
$$

Integration of equation (3.11) gives the result for $\lambda(X)$,

$$
\int_{\lambda(X)}^{\lambda_{L}} \sqrt{\frac{b\left(\lambda^{\prime}\right)}{2}} \frac{\mathrm{d} \lambda^{\prime}}{\Delta\left(\lambda_{L}, \lambda_{R}, \lambda^{\prime}\right)}=\frac{X}{h}
$$

with the requirement for meeting the right end condition

$$
\int_{\lambda_{R}}^{\lambda_{L}} \sqrt{\frac{b\left(\lambda^{\prime}\right)}{2}} \frac{\mathrm{d} \lambda^{\prime}}{\Delta\left(\lambda_{L}, \lambda_{R}, \lambda^{\prime}\right)}=\frac{L}{h} .
$$

This solves the problem of finding post-bifurcated solutions: if we regard the stretch at the center of the neck $\lambda_{L}$ as the control parameter of the solution, then equations (3.12) and (3.14) define $\lambda_{R}\left(\lambda_{L}\right)$, and equation (3.10) defines $n\left(\lambda_{L}\right)$.

For the plots shown in the upcoming figures, we also need the expressions of $\lambda(x)$ and the imposed displacement $\delta$ : by $\lambda=\mathrm{d} x / \mathrm{d} X$, an implicit equation for $\lambda(x)$ is $\int_{\lambda(x)}^{\lambda_{L}} \sqrt{\frac{b\left(\lambda^{\prime}\right)}{2}} \frac{\lambda^{\prime} \mathrm{d} \lambda^{\prime}}{\Delta\left(\lambda_{L}, \lambda_{R}, \lambda^{\prime}\right)}=\frac{x}{h}$, while $\delta=$ $(x(L)-L)$ is given by $\frac{\delta}{h}=\int_{\lambda_{R}}^{\lambda_{L}} \sqrt{\frac{b\left(\lambda^{\prime}\right)}{2}} \frac{\left(\lambda^{\prime}-1\right) \mathrm{d} \lambda^{\prime}}{\Delta\left(\lambda_{L}, \lambda_{R}, \lambda^{\prime}\right)}$.

Because the term inside the second square root in equation (3.12) must be non-negative, it follows that $w(\lambda)$ must be convex on the range $\lambda_{R} \leq \lambda \leq \lambda_{L}$. Given $\lambda_{L}$, the smallest value of $\lambda_{R}$, call it $\lambda_{R}^{*}$, such that the term inside the square root will be non-negative on the range $\lambda_{R}^{*} \leq \lambda \leq \lambda_{L}$ is given by

$$
\frac{\mathrm{d} w}{\mathrm{~d} \lambda}\left(\lambda_{R}^{*}\right)=\frac{w\left(\lambda_{L}\right)-w\left(\lambda_{R}^{*}\right)}{\lambda_{L}-\lambda_{R}^{*}}
$$

The search for $\lambda_{R}$ in terms of $\lambda_{L}$ using (3.14) is therefore limited to $\lambda_{R}^{*} \leq \lambda_{R}<\lambda_{\mathrm{c}}$. Further manipulations of the integrals in equations (3.13-3.14), which are required for numerical accuracy when $\lambda_{R}$ is only slightly larger than $\lambda_{R}^{*}$, are given in Appendix A. 

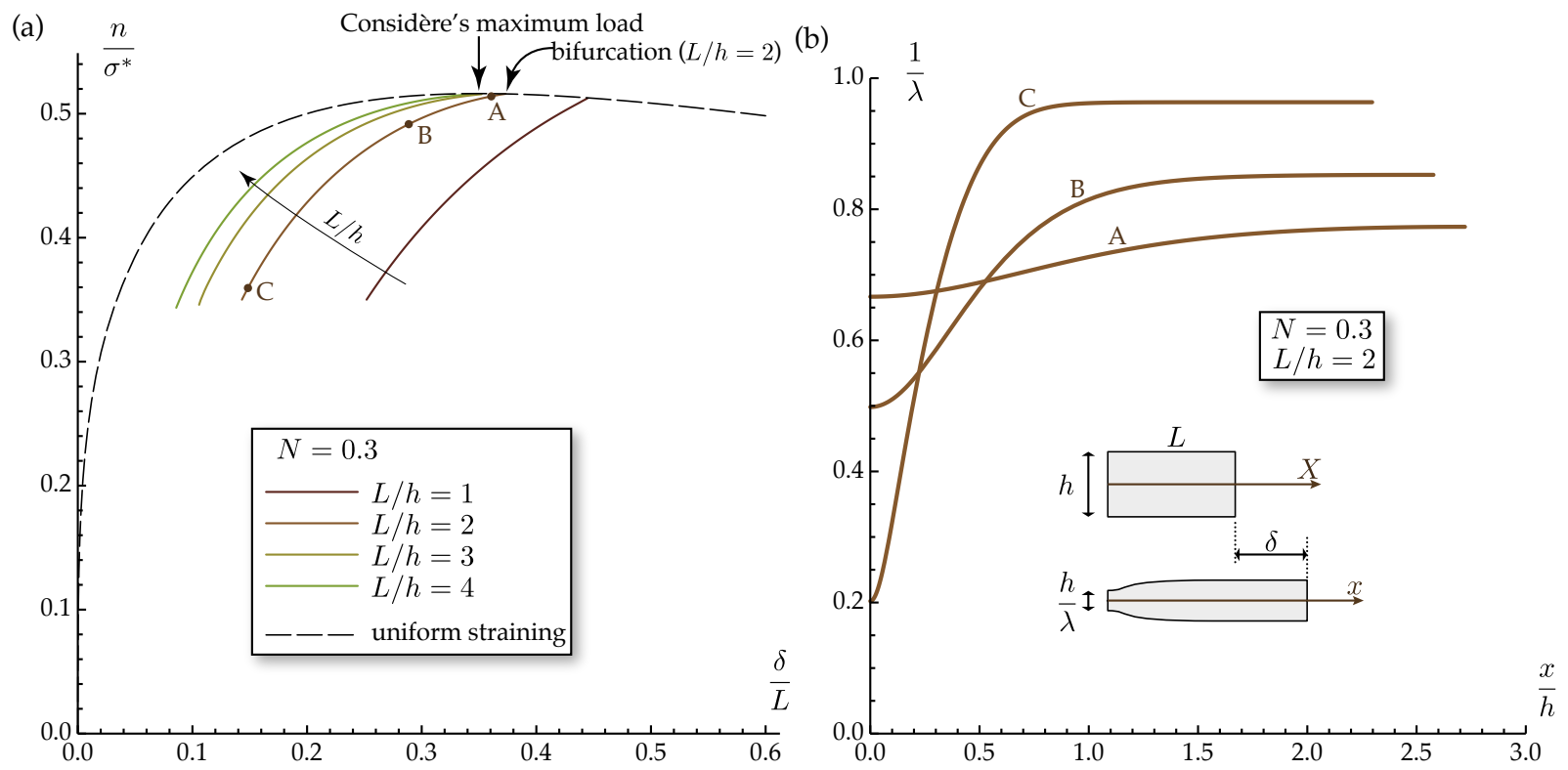

Figure 3: Bifurcated solutions of the boundary value problem (3.8) representing a half-block made up of a nonlinear elastic power-law material with $N=0.3$. (a) Nominal stress as a function of elongation of the half-block for layers with four initial ratios of half-length to thickness, $L / h$. The dashed curve displays the relation for uniform straining with no bifurcation. Solid curves correspond to solutions with a single central neck, obtained by a bifurcation; note that the bifurcation takes place after the load has reached a maximum, and closer and closer to this maximum for slender and slender geometries $(L / h \rightarrow \infty)$. (b) Normalized thickness distribution of the layer, $1 / \lambda(x)$, expressed in terms of the coordinate in the deformed state, $x / h$, for three configurations along the bifurcated branch, corresponding to $\left(\lambda_{L}, n\right)_{A}=(1.5,0.51),\left(\lambda_{L}, n\right)_{B}=(2 ., 0.49)$ and $\left(\lambda_{L}, n\right)_{C}=(5 ., 0.36)$, respectively. The initial half-length to thickness ratio of the layer is $L / h=2$.

As a digression, it can be noted that the bifurcation condition (3.1) emerges directly from (3.14). To see this, expand the right hand expression in (3.14) about $\lambda_{\mathrm{c}}$ using $\lambda_{L}=\lambda_{\mathrm{c}}+\hat{\lambda}_{L}, \lambda_{R}=\lambda_{\mathrm{c}}+\hat{\lambda}_{R}$ and $\lambda=\lambda_{\mathrm{c}}+\hat{\lambda}$. Neglect terms of order $\hat{\lambda}^{3}$ and higher to obtain

$$
\Delta \approx \sqrt{-\frac{1}{2} \frac{\mathrm{d}^{2} w}{\mathrm{~d} \lambda^{2}}\left(\lambda_{\mathrm{c}}\right)} \sqrt{\left(\hat{\lambda}_{L}-\hat{\lambda}\right)\left(\hat{\lambda}-\hat{\lambda}_{R}\right)}
$$

Thus, for small $\hat{\lambda}$, equation (3.14) becomes

$$
\sqrt{\frac{b\left(\lambda_{\mathrm{c}}\right)}{-\frac{\mathrm{d}^{2} w}{\mathrm{~d} \lambda^{2}}\left(\lambda_{\mathrm{c}}\right)}} \int_{\hat{\lambda}_{R}}^{\hat{\lambda}_{L}} \frac{d \hat{\lambda}}{\sqrt{\left(\hat{\lambda}_{L}-\hat{\lambda}\right)\left(\hat{\lambda}-\hat{\lambda}_{R}\right)}}=\frac{L}{h}
$$

The integral evaluates to $\pi$, and thus we recover the bifurcation criterion in equation (3.1).

Solutions generated by numerical integration of the above formulae are presented in figure 3. For $N=0.3$, the maximum load occurs at $\varepsilon_{\mathrm{m}}=0.3$ and $\lambda_{\mathrm{m}}=1.3499$ or, equivalently, at the elongation $\delta / L=0.3499$. The delay beyond the maximum load is evident in figure $3 \mathrm{a}$. This figure also reveals that the post-bifurcation necking behavior of the layer of nonlinear elastic power-law material is highly unstable. Even if the block were stretched in a rigid testing machine that imposed an overall elongation, $\delta$, no bifurcation solution exists at elongations greater than that at the bifurcation point. A block of this material stretched to the bifurcation point would snap dynamically to a state not revealed in figure 3a. The curves plotted in figure 3 a have been terminated when the stretch at the center of the neck attains $\lambda_{L} \sim 5$ because it is unlikely that the approximations used in developing the one-dimensional model are valid for deeper necks. If one does carry the model predictions further, one finds that the extended curves in figure 3a intersect the horizontal axis at an elongation much smaller than $\delta_{\mathrm{c}}$ as $\lambda_{L} \rightarrow \infty$. Thus, the model predicts that a block of this material, 
if stretched to the bifurcation strain, would snap dynamically and "break" when the minimum point of the neck shrinks to zero thickness. For this material, the elastic energy stored in the uniformly strained layer at the bifurcation point is more than sufficient to drive the layer to neck to a point with the elongation at bifurcation imposed. The limiting behavior in figure 3 a for a very slender block, $L / h \rightarrow \infty$, would lie on the curve for uniform straining - except for continuing stretch in the localized neck of width of order $h$ near $X=0$, the rest of the block would unload by uniform straining.

For a block with initial half-length to thickness ratio, $L / h=2$, the shape of the block at three stages after bifurcation is shown in figure $3 \mathrm{~b}$ as a function of the coordinate in the deformed state $x$. The width of the neck decreases as localization proceeds. As a check on the solutions presented in figure 3, they have been verified by an independent numerical solution of the second order boundary-value problem, based directly on equation (3.8), using the library AUTO-07p for numerical continuation (Doedel et al., 2007).

The results of an initial post-bifurcation expansion of the governing differential equation (3.8) about the bifurcation point in the spirit of Koiter (Koiter, 1965; van der Heijden, 2008) can be used to assess the stability at bifurcation and, in particular, to evaluate the initial slope of the load-elongation response at bifurcation seen in figure 3a. Denote the uniform stretch in fundamental uniform solution by $\lambda_{0}$ and the associated nominal stress by $n_{0}=n\left(\lambda_{0}\right)=w^{\prime}\left(\lambda_{0}\right)$ where now $(\cdot)^{\prime}=\mathrm{d}(\cdot) / \mathrm{d} \lambda$. The extension of the ends in the fundamental solution is $\delta / L=\lambda_{0}-1$. The bifurcation mode is $\lambda_{1}=\cos (\pi X / L)$ with $\lambda_{\mathrm{c}} \operatorname{satisfying}(3.1)$. With $\xi$ as the amplitude of the bifurcation mode, the expansion about $\lambda_{\mathrm{c}}$ has the form

$$
\begin{aligned}
\lambda(X) & =\lambda_{0}+\xi \lambda_{1}(X)+\xi^{2} \lambda_{2}(X)+\cdots \\
n & =w^{\prime}\left(\lambda_{0}\right)+\xi^{2} n_{2}+\xi^{4} n_{4} \cdots \\
\lambda_{0} & =\lambda_{\mathrm{c}}+\xi^{2} a+\xi^{4} c+\cdots
\end{aligned}
$$

Regard $\delta$ as the prescribed load parameter. Then, $\int_{0}^{L} \lambda_{i} \mathrm{~d} X=0$ for $i=1,2, \cdots$ because the fundamental solution satisfies $\delta / L=\lambda_{0}-1$. In addition, the mode amplitude is uniquely defined if $\int_{0}^{L} \lambda_{i} \lambda_{1} \mathrm{~d} X=0$ for $i>1$. The important terms for the present study are found to be

$$
\begin{aligned}
\lambda_{2}(X) & =\lambda_{2}^{0} \cos \frac{2 \pi X}{L} \quad \text { where } \lambda_{2}^{0}=-\frac{w_{\mathrm{c}}^{\prime \prime \prime}+3\left(\frac{\pi h}{L}\right)^{2} b_{\mathrm{c}}^{\prime}}{4\left(w_{\mathrm{c}}^{\prime \prime}+4\left(\frac{\pi h}{L}\right)^{2} b_{\mathrm{c}}\right)} \\
n_{2} & =\frac{1}{4}\left(w_{\mathrm{c}}^{\prime \prime \prime}+\left(\frac{\pi h}{L}\right)^{2} b_{\mathrm{c}}^{\prime}\right) \\
a & =-\frac{1}{32 n_{2}}\left(4 \lambda_{2}^{0} w_{\mathrm{c}}^{\prime \prime \prime}+w_{\mathrm{c}}^{\prime \prime \prime \prime}+2\left(\frac{\pi h}{L}\right)^{2}\left(6 \lambda_{2}^{0} b_{\mathrm{c}}^{\prime}+b_{\mathrm{c}}^{\prime \prime}\right)\right)
\end{aligned}
$$

where $w_{\mathrm{c}}^{\prime \prime \prime}=\frac{\mathrm{d}^{3} w}{\mathrm{~d} \lambda^{3}}\left(\lambda_{\mathrm{c}}\right)$ denotes the third derivative with respect to $\lambda$ evaluated at $\lambda_{\mathrm{c}}$, and similarly for $b_{\mathrm{c}}^{\prime}$ etc. The initial slope of the load-elongation behavior of bifurcated solution is given by

$$
\frac{n-n_{\mathrm{c}}}{\frac{1}{L}\left(\delta-\delta_{\mathrm{c}}\right)}=w_{\mathrm{c}}^{\prime \prime}+\frac{n_{2}}{a} .
$$

The initial slope is plotted in figure 4 for several values of $N$ for the power-law material. The values for $N=0.3$ are in agreement with the initial slopes of the curves in figure 3a. In addition, for $N=0.2$ and 0.3 over the range plotted in figure 4 , the initial slope is positive, consistent with the unstable behavior discussed in connection with figure 3a. For $N=0.1$, however, the denominator $a$ in equation (3.18) passes through zero at $L / h=1.6$ : as a result the initial slope becomes negative for $L / h<1.6$, and the initial post-bifurcation behavior becomes stable under prescribed elongation. Nevertheless, except possibly for very stocky blocks having small $N$, the block of power-law material has a highly unstable post-bifurcation behavior under prescribed elongation. This finding is in agreement with an exact two-dimensional plane strain initial post-bifurcation analysis carried out for a rectangular block of Blatz-Ko material by Triantafyllidis et al. (2007). The Blatz-Ko material also exhibits a maximum nominal stress in plane strain tension and 


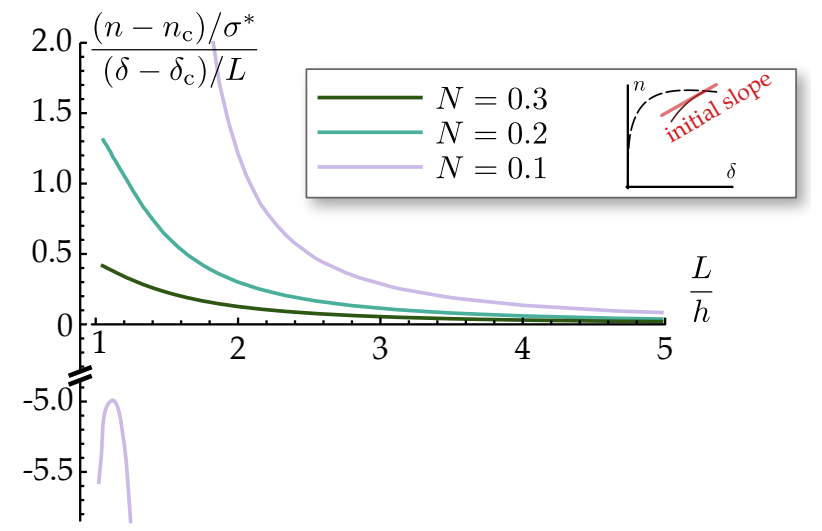

Figure 4: The initial slope of the post-bifurcation load-elongation behavior for the block of nonlinear power-law material, as obtained by the initial post-bifurcation analysis in equation (3.18).

undergoes necking. The block has the same boundary conditions employed in the present study, and the authors perform a Koiter-type analysis to evaluate the initial curvature of the relation of elongation to mode amplitude, analogous to $a$ in (3.16). The block of Blatz-Ko material is unstable at bifurcation under prescribed elongation for all values of the initial aspect ratio considered by Triantafyllidis et al. (2007).

\section{Application to elastic-plastic materials}

In the context of necking, the primary difference between a metal and a nonlinear elastic material (such as that considered in the previous section) is the response of the material when the stretch switches from increasing to decreasing - elastic unloading in plasticity terminology. A metal deformed into the plastic range in tension responds with a stiff linear elastic response as soon as the stretch rate is reversed. By contrast, the unloading response of the nonlinear elastic material is reversible and highly compliant, returning the stored energy as the load drops. The difference between the two unloading responses is responsible for the stable necking behavior observed in most tensile test of metals, at least in the early stages of necking, and in the highly unstable necking behavior seen in the previous section for nonlinear elastic materials. In a metal in uniaxial stressing, the unloading response is taken to be $\dot{\sigma}=E \dot{\varepsilon}$ where the over-dot is the standard notation for an increment in time-independent plasticity and $E$ is Young's modulus. The ratio of the incremental tangent modulus for loading to the Young's modulus at the maximum load is $E_{\mathrm{t}}^{\mathrm{m}} / E=\sigma_{\mathrm{m}} / E$, by Considère's criterion (see $\S 3.1$ ), which is typically less than $10^{-2}$ for metals. Thus, it will be seen that, except for very slender blocks and bars, Young's modulus itself has little influence on the necking response - effectively the material becomes rigid when it unloads. The energy density of the material is first modified to account for the stiff unloading response in Section 4.1, and the one-dimensional model is used to analyze the block of elastic-plastic material in Section 4.2. The transition from stability to instability of necking of very slender blocks of elastic-plastic material under prescribed elongation is analyzed in Section 4.3. In Section 4.4, we calculate a coefficient of stress reduction at the center neck by the one-dimensional model, and show that it agrees with that proposed by Bridgman (1952) up to moderately large necking amplitudes.

\subsection{Extending the one-dimensional model to account for elastic unloading}

A nonlinear elastic constitutive model is commonly used to model the plastic deformation of metals under conditions when the strains increase monotonically and proportionally. In plasticity theory, a constitutive model in this class is called a deformation theory. As an approximation for metals deforming under necking conditions in plane strain, we will employ the nonlinear elastic model (3.6) introduced in the previous section to represent the response to a tension test in the loading range when the stretch increases. Within the framework of the one-dimensional reduction for the necking problem, the criterion for transitioning from 
plastic loading to elastic unloading is the switch from $\dot{\lambda}>0$ to $\dot{\lambda}<0$ at a given material point $X$. At any particular time in the loading history, we denote by $X_{\mathrm{u}}$ the material position of the unloading front, which is such that $\dot{\lambda}\left(X_{\mathrm{u}}\right)=0$. The corresponding stretch is denoted by $\lambda_{\mathrm{u}}\left(X_{\mathrm{u}}\right)$, and corresponds to the maximum value of stretch attained at that material point. Further reversals of stretch-rate with plastic reloading following elastic unloading does not occur in the problems considered in this paper and will not be characterized. Unloading starts far from the center of the neck at the end at $X=L$. As a result, at any given time in the loading history the unloading region is $\left[X_{\mathrm{u}}, L\right]$ and the function $\lambda_{\mathrm{u}}$ is defined on the interval $\left[X_{\mathrm{u}}, L\right]$. As the unloading front propagates in the negative $X$ direction, $X_{\mathrm{u}}$ decreases, and the function $\lambda_{\mathrm{u}}$ gets extended 'to the left'.

For both the plane strain problem and the axisymmetric problem, $\dot{\sigma}=\dot{n} \lambda+n \dot{\lambda} \approx \dot{n} \lambda$, where the term $n \dot{\lambda}$ is neglected in the linear elastic unloading response because it is of order $\sigma / E$ compared to $\dot{n} \lambda$. Thus, for uniform straining, $\dot{\sigma}=E \dot{\varepsilon}$ can be rewritten as $\dot{n}=\left(E / \lambda^{2}\right) \dot{\lambda}$. Because the change in stretch during unloading is very small, this is further approximated as $\dot{n}=\left(E / \lambda_{\mathrm{u}}^{2}\right) \dot{\lambda}$. With $w_{\mathrm{u}}(\lambda)$ denoting the energy under uniform stretch for the unloading response and noting that $\dot{n}=\frac{\mathrm{d}^{2} w_{\mathrm{u}}(\lambda)}{\mathrm{d} \lambda^{2}} \dot{\lambda}$, we identify $\frac{\mathrm{d}^{2} w_{\mathrm{u}}(\lambda)}{\mathrm{d} \lambda^{2}}=E / \lambda_{\mathrm{u}}^{2}$ such that the Taylor expansion of the unloading strain energy reads

$$
w_{\mathrm{u}}(\lambda)=w\left(\lambda_{\mathrm{u}}\right)+n_{\mathrm{u}}\left(\lambda-\lambda_{\mathrm{u}}\right)+\frac{E}{2 \lambda_{\mathrm{u}}^{2}}\left(\lambda-\lambda_{\mathrm{u}}\right)^{2} \quad \text { for } \lambda<\lambda_{\mathrm{u}} .
$$

Here, $w\left(\lambda_{\mathrm{u}}\right)=w_{\mathrm{u}}\left(\lambda_{\mathrm{u}}\right)$ and $n_{\mathrm{u}}=\frac{\mathrm{d} w\left(\lambda_{\mathrm{u}}\right)}{\mathrm{d} \lambda}=\frac{\mathrm{d} w_{\mathrm{u}}\left(\lambda_{\mathrm{u}}\right)}{\mathrm{d} \lambda}$ as both the strain energy and the nominal stress are continuous at the onset of unloading.

Observe that the expansion in equation (4.1) differs from that of the original strain energy $w(\lambda)$ (representing the loading phase) through the quadratic term only. This suggests a modified definition of the strain energy density, which captures both loading and unloading:

$$
w(\lambda)+\frac{\alpha}{2} \frac{E}{\lambda_{\mathrm{u}}^{2}}\left(\lambda-\lambda_{\mathrm{u}}\right)^{2}
$$

where

$$
\alpha= \begin{cases}0 & \text { for } \dot{\lambda}>0 \text { (loading) } \\ 1 & \text { for } \lambda \leq \lambda_{\mathrm{u}} \text { (unloading) }\end{cases}
$$

For uniform stretching, the modified energy density coincides with the original one $w(\lambda)$ in the loading regime; in the unloading regime it differs from $w_{\mathrm{u}}(\lambda)$ in (4.1) with an error of order $\sigma\left(\lambda-\lambda_{\mathrm{u}}\right)^{2}$, which is of order $\sigma / E \ll 1$ compared to $\left(E / \lambda_{\mathrm{u}}^{2}\right)\left(\lambda-\lambda_{\mathrm{u}}\right)^{2}$. When unloading sets in, $\frac{1}{2}\left(E / \lambda_{\mathrm{u}}^{2}\right)\left(\lambda-\lambda_{\mathrm{u}}\right)^{2}$ dominates changes in $w(\lambda)$ for small changes of the stretch from $\lambda_{\mathrm{u}}$.

Accordingly, the one-dimensional strain energy $W$ in equation (1.2) becomes, for the elastic-plastic material,

$$
W_{\mathrm{ep}}=h \int_{0}^{L}\left[w(\lambda)+\frac{h^{2}}{2} b(\lambda)\left(\frac{\mathrm{d} \lambda}{\mathrm{d} X}\right)^{2}+\frac{\alpha}{2} \frac{E}{\lambda_{\mathrm{u}}^{2}}\left(\lambda-\lambda_{\mathrm{u}}\right)^{2}\right] \mathrm{d} X
$$

where $w(\lambda)$ and $b(\lambda)$ retain the definition used in the loading regime (recall that the subscript 0 in the notation $w_{0}(\lambda)$ for the strain energy in uniform straining has been dropped since Section 3 onwards). In the limit in which the solid is taken to be rigid upon unloading $(E \rightarrow \infty)$, the unloading stretch is locked at $\lambda=\lambda_{\mathrm{u}}$. The second-gradient term $b(\lambda) \lambda^{\prime 2}$ could also be modified in the unloading regime but it does not play an important role and, thus, it has not been altered. The energy for the round bar in equation (1.4) is modified similarly.

The solution for the elastic-plastic material is incremental. In each increment, the load $n$ is incremented by $\dot{n}$ and the increment $\dot{\lambda}(X)$ is calculated by solving the equilibrium equation corresponding to the energy functional $W_{\mathrm{ep}}+E_{\mathrm{p}}$, linearized near the current configuration; the unloading boundary $X_{\mathrm{u}}$ is also updated using the condition $\dot{\lambda}\left(X_{\mathrm{u}}\right)=0$. The reader is referred to Appendix B for details. As earlier in equation (3.7), $E_{\mathrm{p}}$ is the potential energy associated with the external load. 

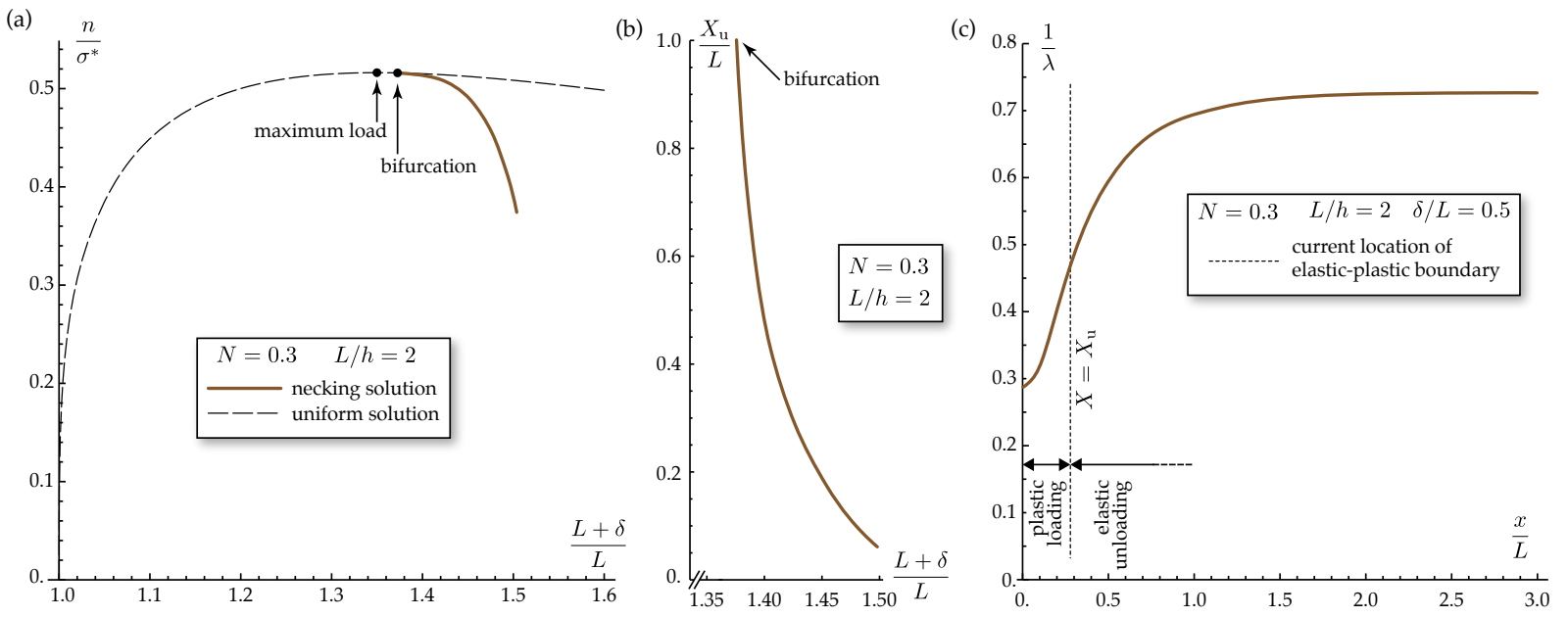

Figure 5: Necking solution for a block of elastic-plastic material with $N=0.3, \sigma^{*} / E=0.003$ and $L / h=2$. (a) Nominal stress (load) vs. overall imposed stretch. Upon bifurcation, the plastic loading response occurs throughout the entire block: the bifurcation load is identical to that of a nonlinearly elastic material shown in figure 3a. (b) Location $X_{\mathrm{u}}$ of the boundary between the plastically loading region, $X<X_{\mathrm{u}}$, and elastically unloading region, $X \geq X_{\mathrm{u}}$, as a function of the overall stretch. (c) Shape of the neck at the elongation $\delta / L=0.5$ as measured by $1 / \lambda(x)$ where $x$ is the distance along the axis in the current deformed state.

\subsection{Necking of elastic-plastic blocks}

As just emphasized, the necking behavior of a block elastic-plastic material subject to prescribed elongation $\delta$, is intrinsically incremental in that the boundary between plastically loading and elastic unloading regions starts at the right end of the block and moves continuously towards the center. The solution procedure must track this boundary and the spatial distribution of $\lambda_{\mathrm{u}}(X)$. A numerical scheme is essential for solving the governing equations of the one-dimensional model. The algorithm used in this paper, which is described in Appendix B, makes use of a finite element approximation of $W_{\mathrm{ep}}+E_{\mathrm{p}}$, with $W_{\mathrm{ep}}$ defined in (4.3) and $E_{p}=-h n \int_{0}^{L} \lambda \mathrm{d} X$.

The load-elongation curve for the block is shown in figure 5 a for $N=0.3, \sigma^{*} / E=0.003$ and an initial half-length to thickness, $L / h=2$. The location of the boundary between the loading and unloading regions, $X_{\mathrm{u}}$, is shown in figure $5 \mathrm{~b}$, and the through-thickness stretch distribution, $1 / \lambda(x)$, at the elongation $\delta / L=0.5$ is plotted in figure 5c. In this example, the neck develops under increasing elongation implying stable behavior under prescribed elongation. The contrast with the block of nonlinear elastic material in figure 3a is striking. Whereas unloading in the region outside the neck for the nonlinear elastic material supplies energy to drive the necking deformation, the switch to the stiff elastic response upon unloading in the elastic-plastic model significantly reduces the energy released by the unloaded segment. The plot in figure 5 is representative of what is measured in an experiment or what one would obtain from a detailed finite element computation for an elastic-plastic block. Prescribed elongation is equivalent to tensile testing in a so-called rigid machine which imposes the separation of the specimen ends. The computation in figure 5 has not been carried to the point where the overall stretch reaches a maximum, which would occur at $(L+\delta) / L \approx 1.5$ : this would be the instability point under prescribed elongation. Of course, the block would become unstable at maximum load if the block was loaded by dead weight, i.e. by a prescribed force.

As is generally the case for bifurcation in elastic-plastic solids (Hill, 1961), the lowest bifurcation occurs with the plastic loading response taking place throughout the block. As a consequence, the stretch at bifurcation $\lambda_{c}$ is still given by equation (3.1). The bifurcation mode is a superposition of the eigenmode $\cos (\pi X / L)$ and an increment of uniform stretch. However, elastic unloading begins at bifurcation at the right end of the block and the region of unloading spreads towards the center of the neck, as seen in figure $5 \mathrm{~b}$. The spread of the unloading region is remarkably rapid. As seen in figure 5b, the loading/unloading boundary has reached the mid-point between the center and the end of the block at an additional overall elongation 
(a)

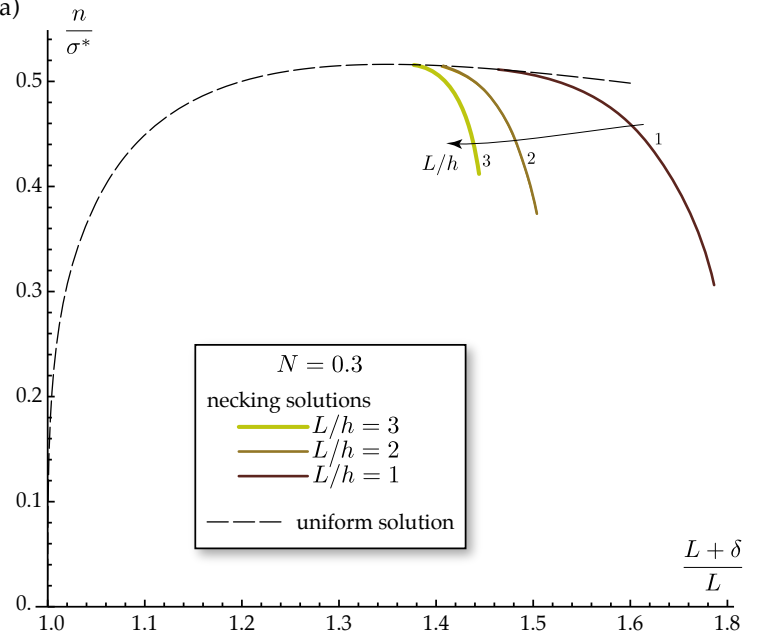

(b)

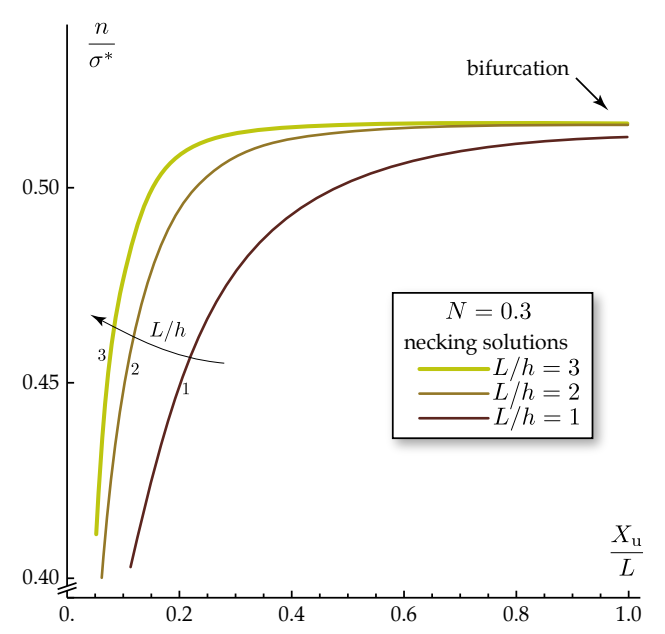

Figure 6: a) Load-overall stretch response and b) load versus location of the boundary between the loading and unloading regions for three ratios of the initial half-length to thickness. The block material is elastic-plastic with $N=0.3$ and $\sigma^{*} / E=0.003$.

of only about $1 \%$ and a very slight drop of nominal stress $n$. At the elongation $\delta / L=0.5$ in figure 5 c, the boundary has reached $x / L=0.28$ in the deformed state $\left(X_{\mathrm{u}} / L \approx 0.12\right.$ in the undeformed coordinate) and the thickness of the neck at the center is $\approx 0.29 \mathrm{~h}$. Because unloading sweeps across the block so rapidly and because the unloading response is so stiff, the thickness of the right half of the block in figure $5 \mathrm{c}$ is nearly that at bifurcation.

The effect of the initial aspect ratio of the block $L / h$ is illustrated in figure 6 . Each block is initially stable, but the more slender the block the less overall stretch that can be imposed on the block before it becomes unstable under prescribed elongation. Figure $6 \mathrm{~b}$ again demonstrates the exceptionally rapid spread of the unloading region from the end of the block immediately after bifurcation. For the block with initial aspect ratio $L / h=3$ continuing plastic deformation immediately localizes to a necking region whose length is on the order of $h$.

Because localization in the neck occurs within a region of order $h$ from the center of the block with nearly all the plastic straining taking place within this region, it is insightful to plot results for the example in figure 6 as $n / \sigma^{*}$ versus the additional average stretch after bifurcation in the region $0 \leq X \leq h,\langle\lambda\rangle_{h} \equiv x(h) / h-\lambda_{\mathrm{c}}$, as in figure 7. This figure reveals that for blocks with aspect ratios satisfying $L / h \geq 2$ the deformation within the region $(0 \leq X \leq h)$ becomes independent of $L / h$. Localization occurs so rapidly after bifurcation that nearly all the post-bifurcation plastic deformation occurs within the region in the localized neck. Moreover, the curves in figure 7 are essentially independent of $\sigma^{*} / E$ over the range $10^{-3} \leq \sigma^{*} / E \leq 10^{-2}$ covering the strength to elasticity modulus ratio of nearly all metals. For the approximate analysis of slender blocks in the next sub-section, denote the relation between $n / \sigma^{*}$ and $\langle\lambda\rangle_{h}$ in figure 7 for $L / h \geq 2$ by $n / \sigma^{*}=F_{N}\left(\langle\lambda\rangle_{h}\right)$. Specifically, for $N=0.3$, the curve in figure 7 is accurately approximated by

$$
F_{N=0.3}\left(\langle\lambda\rangle_{h}\right)=0.5160-0.6694\langle\lambda\rangle_{h}^{2}-22.131\langle\lambda\rangle_{h}^{4} \quad \text { for }\langle\lambda\rangle_{h}<0.25
$$

\subsection{Necking stability of very slender blocks of elastic-plastic material under prescribed elongation}

While elastic unloading has almost no influence of necking stability of relatively stocky blocks, it does determine the stability under prescribed elongation for sufficiently slender blocks. When the block is slender enough the energy released from elastic unloading can drive the localization, analogous to what was seen for stocky blocks of nonlinear elastic material. For large aspect ratio blocks it is possible to exploit the localization behavior discussed above by linking the response in the central necking section to the elastic unloading response outside that section. This approach provides an approximate, but highly accurate, 


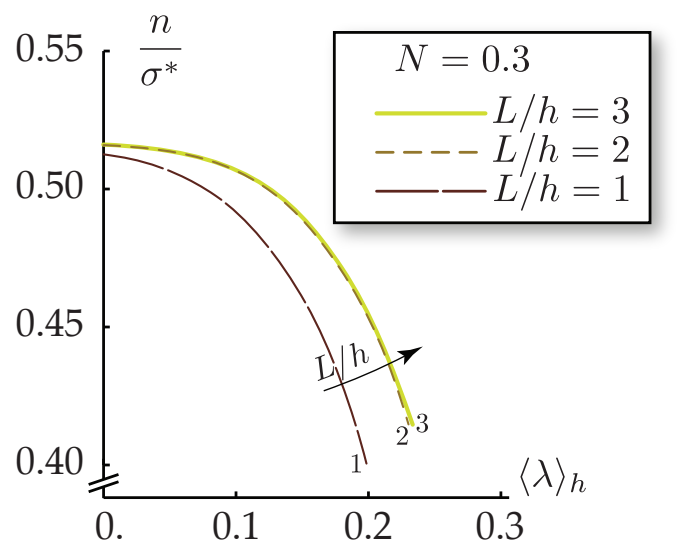

Figure 7: Normalized load $n / \sigma^{*}$ versus the additional average stretch after bifurcation in the region $0 \leq X \leq h,\langle\lambda\rangle_{h} \equiv$ $x(h) / h-\lambda_{\mathrm{c}}$. The block material is elastic-plastic with $N=0.3$ and $\sigma^{*} / E=0.003$. As seen above, this relation is essentially independent of the initial aspect ratio if $L / h \geq 2$, and these curves are virtually unchanged when $\sigma^{*} / E$ is increased from $10^{-3}$ to $10^{-2}$.

analysis of the effect of block aspect ratio on the necking response after bifurcation. Because $n$ is uniform along the entire block it follows that after bifurcation $n$ must be the same in the necking region, $0 \leq X \leq h$, and in the remaining region, $h \leq X \leq L$, which is assumed to undergo unloading. Using the equality $\dot{n}=\frac{n}{\lambda_{\mathrm{u}}^{2}} \dot{\lambda}$ established in $\S 4.1$, this condition requires

$$
\frac{n}{\sigma^{*}}=F_{N}\left(\langle\lambda\rangle_{h}\right)=\frac{n_{\mathrm{c}}}{\sigma^{*}}+\frac{E}{\sigma^{*}} \frac{1}{\lambda_{\mathrm{c}}^{2}} \frac{\delta-\delta_{\mathrm{c}}-\langle\lambda\rangle_{h} h}{(L-h)}
$$

which can be re-expressed as

$$
\frac{\delta-\delta_{\mathrm{c}}}{h}=\frac{\sigma^{*} L}{E h} \lambda_{\mathrm{c}}^{2}\left(1-\frac{h}{L}\right)\left[F_{N}\left(\langle\lambda\rangle_{h}\right)-F_{N}(0)\right]+F_{N}\left(\langle\lambda\rangle_{h}\right)
$$

This expression is expected to be accurate for blocks having $L / h>2$. The only dimensionless parameter expressing the role of elasticity is $\frac{\sigma^{*} L}{E h}$, and $\left(1-\frac{h}{L}\right)$ is nearly unity for very slender blocks.

The effect of the block aspect ratio is illustrated in figure 8 for the same example under consideration $\left(N=0.3\right.$ and $\left.\sigma^{*} / E=0.003\right)$ but now with a wide range of aspect ratios including values as large as $\frac{L}{h}=200$ corresponding to $\frac{\sigma^{*} L}{E h}=0.6$. When $L / h$ is large $\left(i . e . \frac{\sigma^{*} L}{E h} \sim 1\right)$, only a very small additional elongation can be imposed on the wire beyond bifurcation before it becomes unstable under prescribed end displacement. The block with $L / h=200$ would become unstable and snap dynamically when the additional end displacement is only $\approx 0.06 h$. For practical purposes, one can say the block will become unstable immediately upon attaining the Considère strain if $\frac{\sigma^{*} L}{E h} \sim 1$. For round bars with large aspect ratio (e.g. piano wires), this unstable behavior has been referred to as the "piano tuner's problem". By turning the end peg of the piano wire, the tuner prescribes the elongation and increases the tension of the wire. If the tuner turns beyond the instability point the wire snaps dynamically leaving a nicely formed neck at some point along the wire. In conclusion, the highly unstable behavior seen in connection with the nonlinear elastic material is also observed when the material displays linear elastic unloading if the aspect ratio is sufficiently large.

\subsection{Effective stress reduction at the center of the neck and the connection to the Bridgman approximation}

As Bridgman (1952) noted, the curvature of a round bar at the center of the neck reduces the Mises effective stress relative to the axial component of the Cauchy stress. As a result, the strain at the center of the neck is less than it would be for a uniform bar at the same Cauchy stress. Bridgman's formulae for this reduction are used to this day to extract the uniaxial stress-strain behavior from test data for specimens 


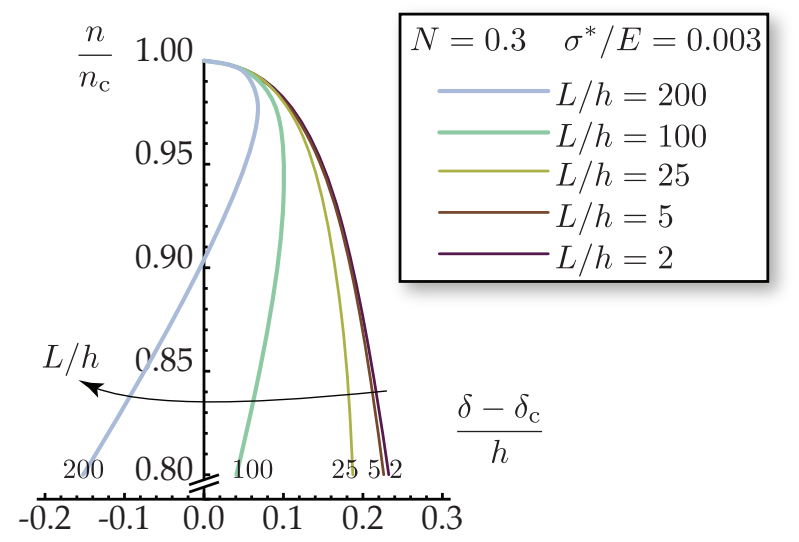

Figure 8: The load-elongation behavior based on equation (4.6) for slender blocks of elastic-plastic material with $N=0.3$ and $\sigma^{*} / E=0.003$. The single dimensionless parameter $\sigma^{*} L /(E h)$ controls the stability for slender blocks.

that are strained well past the onset of necking. Similar reductions are predicted by the one-dimensional model. We begin by considering the block and then make contact with Bridgman's result for the round bar.

By equations (3.8a) and (1.2), the nominal stress $n$ in the block is independent of $X$ and reads, when evaluated at the center of the neck,

$$
n=\frac{\mathrm{d} w}{\mathrm{~d} \lambda}\left(\lambda_{\mathrm{ct}}\right)-h^{2} b\left(\lambda_{\mathrm{ct}}\right) \frac{\mathrm{d}^{2} \lambda}{\mathrm{d} X^{2}}\left(\lambda_{\mathrm{ct}}\right)=n_{0}\left(\lambda_{\mathrm{ct}}\right)\left(1-\frac{h^{2}}{12 \lambda_{\mathrm{ct}}^{5}} \frac{\mathrm{d}^{2} \lambda}{\mathrm{d} X^{2}}\left(\lambda_{\mathrm{ct}}\right)\right),
$$

where $\lambda_{\text {ct }}=\lambda(0)$ is the stretch at the center of the neck and the 'effective nominal stress' $n_{0}(\lambda) \equiv \frac{\mathrm{d} w}{\mathrm{~d} \lambda}$ is the nominal stress if the stretch were uniform and equal to $\lambda_{\mathrm{ct}}$. Similarly, the 'effective true stress' is $\sigma_{0}\left(\lambda_{\mathrm{ct}}\right)$, where $\sigma_{0}(\lambda)=\lambda n_{0}(\lambda)$ is the true stress at uniform stretch $\lambda$. The average true stress at the center of the neck is $\sigma_{\mathrm{ct}}=\lambda_{\mathrm{ct}} n$. In a tension test to measure the stress-stretch curve of the metal, it is the function $\sigma_{0}(\lambda)$ that is sought. Noting that $\sigma_{0}\left(\lambda_{\mathrm{ct}}\right) / \sigma_{\mathrm{ct}}=n_{0}\left(\lambda_{\mathrm{ct}}\right) / n$, it follows from (4.7) that at the center of the neck

$$
\frac{\sigma_{0}\left(\lambda_{\mathrm{ct}}\right)}{\sigma_{\mathrm{ct}}}=\left(1-\frac{h^{2}}{12 \lambda_{\mathrm{ct}}^{5}} \frac{\mathrm{d}^{2} \lambda}{\mathrm{d} X^{2}}\left(\lambda_{\mathrm{ct}}\right)\right)^{-1}=\left(1+\frac{\bar{h}(0)}{12} \frac{\mathrm{d}^{2} \bar{h}}{\mathrm{~d} x^{2}}(0)\right)^{-1}
$$

where $\bar{h}(x)=h / \lambda(x)$ is the deformed thickness variation in terms of the coordinate $x$ in the deformed state and $\mathrm{d}^{2} \bar{h} / \mathrm{d} x^{2}$ is the curvature of the outer boundary measured in the axial direction and at the center of the neck. In a tensile test, $\sigma_{0}\left(\lambda_{\text {ct }}\right)$ cannot be measured directly once necking starts. However, one can measure the average true stress $\sigma_{\text {ct }}$ as a function of the stretch at the center of the neck, as well as the thickness $\bar{h}$ and the curvature $\mathrm{d}^{2} \bar{h} / \mathrm{d} x^{2}$. The desired relation $\sigma_{0}(\lambda)$ can then be obtained from (4.8). Examples illustrating the direct calculation of the ratio $\sigma_{0}\left(\lambda_{\text {ct }}\right) / \sigma_{\text {ct }}$ using (4.8) based on the simulations in Section 4.2 are shown in figure 9 .

The corresponding formula for the round bar derived from (1.4) is

$$
\frac{\sigma_{0}\left(\lambda_{\mathrm{ct}}\right)}{\sigma_{\mathrm{ct}}}=\left(1-\frac{D^{2}}{32 \lambda_{\mathrm{ct}}^{4}} \frac{\mathrm{d}^{2} \lambda}{\mathrm{d} X^{2}}\left(\lambda_{\mathrm{ct}}\right)\right)^{-1}=\left(1+\frac{\bar{R}(0)}{4} \frac{\mathrm{d}^{2} \bar{R}}{\mathrm{~d} x^{2}}(0)\right)^{-1}=\frac{1}{1+\frac{\rho}{4}},
$$

where $\bar{R}(x)=D /(2 \sqrt{\lambda(x)})$ is the radius of the bar in the deformed state, and $\rho=\bar{R}(0) \frac{\mathrm{d}^{2} \bar{R}}{\mathrm{~d} x^{2}}(0)$ is the ratio of the radius $\bar{R}(0)$ at the center of the neck to the axial radius of curvature of the lateral surface there. Bridgman's result for this ratio is (see equation [1-13] in his book)

$$
\left.\frac{\sigma_{0}\left(\lambda_{\mathrm{ct}}\right)}{\sigma_{\mathrm{ct}}}\right|_{\mathrm{B}}=\frac{1}{\left(1+\frac{2}{\rho}\right) \ln \left(1+\frac{\rho}{2}\right)}
$$




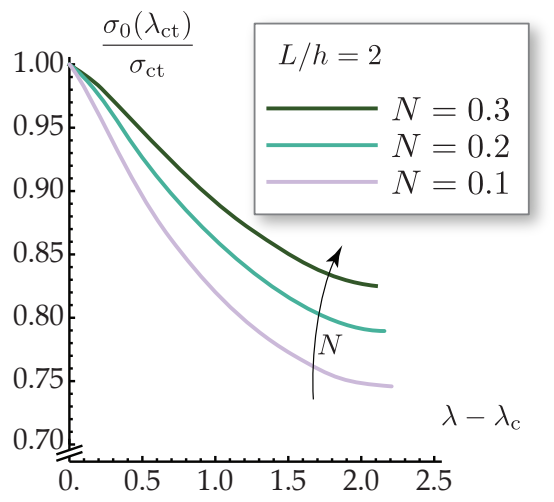

Figure 9: Ratio of the effective true stress to the average true stress as a function of the additional stretch after bifurcation at the center of the neck as predicted by the one-dimensional model of the block for three values of the strain hardening exponent. The effective true stress is the stress which would occur at the stretch $\lambda$ if the block were constrained to deform uniformly.

(a) $\frac{\sigma_{0}\left(\lambda_{\mathrm{ct}}\right)}{\sigma_{\mathrm{ct}}}$

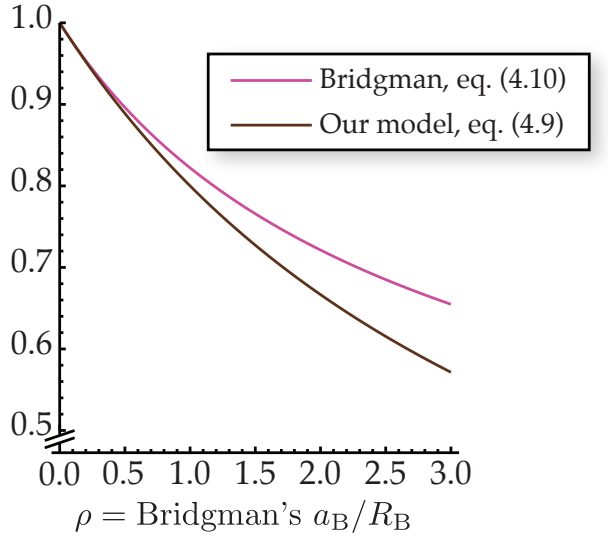

(b)

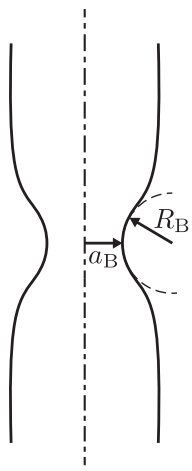

Figure 10: (a) Comparison between Bridgman's 'correction factor' and the result from the one-dimensional reduction for a round bar, expressed in terms of the ratio $\rho$ of the cross-sectional radius to the radius of curvature at the center of neck. (b) Using Bridgman's notation, $\rho=a_{\mathrm{B}} / R_{\mathrm{B}}$.

where, using his own notation, $\rho=\frac{a_{\mathrm{B}}}{R_{\mathrm{B}}}, a_{\mathrm{B}}$ is the radius at the center of the neck $(\bar{R}(0)$ in our notation) and $R_{\mathrm{B}}$ the radius of curvature there $\left(1 / \frac{\mathrm{d}^{2} \bar{R}}{\mathrm{~d} x^{2}}(0)\right.$ in our notation).

A comparison of (4.9) and (4.10) is shown in figure 10. The two formulae agree exactly to order $\rho=$ $a_{\mathrm{B}} / R_{\mathrm{B}}$. Our one-dimensional model therefore provides a rigorous justification for Bridgman's formula in the limit $\rho \ll 1$.

It is interesting to contrast the prediction of our model with that based on Coleman-Newman's upper bound construction, which includes a spurious factor $\frac{\lambda^{3}}{\lambda^{3}-1}$, see (2.32b). Adapting the argument in equation (4.9), we find that the upper bound construction predicts a stress reduction factor

$$
\left.\frac{\sigma_{0}\left(\lambda_{\mathrm{ct}}\right)}{\sigma_{\mathrm{ct}}}\right|_{\mathrm{C}}=\left(1-\frac{D^{2}}{32 \lambda_{\mathrm{ct}}^{4}} \frac{\lambda_{\mathrm{ct}}^{3}}{\lambda_{\mathrm{ct}}^{3}-1} \frac{\mathrm{d}^{2} \lambda}{\mathrm{d} X^{2}}\left(\lambda_{\mathrm{ct}}\right)\right)^{-1}=\left(1+\frac{\lambda_{\mathrm{ct}}^{3}}{\lambda_{\mathrm{ct}}^{3}-1} \frac{\bar{R}(0)}{4} \frac{\mathrm{d}^{2} \bar{R}}{\mathrm{~d} x^{2}}(0)\right)^{-1}=\frac{1}{\frac{\lambda_{\mathrm{ct}}^{3}}{\lambda_{\mathrm{ct}}^{3}-1}\left(1+\frac{\rho}{4}\right)} .
$$

Coleman-Newman's prediction for the stress reduction is typically off by a factor $\sim 40 \%$ (the typical value of the spurious factor in the denominator is 1.81, as noted earlier). In addition, the spurious factor brings in an additional dependence on the stretch $\lambda_{\mathrm{ct}}$ and the stress reduction is not a function of $\rho$ only -in contrast with Bridgman's formula (4.9) and ours (4.10).

This shows that Bridgman's formula captures a subtle feature of necking, revealed by the asymptotically exact 1D-model: the existence of a simple relation between the stress reduction factor $\sigma_{0}\left(\lambda_{\mathrm{ct}}\right) / \sigma_{\mathrm{ct}}$ and the 
geometric parameter $\rho$. The less accurate upper-bound construction of Coleman-Newman fails to capture this feature.

\section{Conclusions and suggestions for further research}

A one-dimensional model has been derived for the necking analysis of rectangular blocks and round bars. It is carried out within the framework of nonlinear elastic solids but it has been extended for applications to elastic-plastic solids. By incorporating gradients of the strains that arise during necking, the model is capable of capturing the highly nonlinear localization process that accompanies necking. Bifurcation from the uniform state into the necking mode is accurately reproduced by the model. Moreover, comparison with the widely employed Bridgman formula for extracting uniaxial stress-strain behavior from data measured from a necked specimen suggests that the one-dimensional model remains accurate for relatively deep necks.

The dimensional reduction carried out in this paper takes into account the curvature of the material lines initially normal to the axis of the slab or bar. This turned out to be crucial for the correctness of the dimensional reduction, as it is otherwise impossible to relax the shear stress at the free boundaries. This curvature effect has been ignored in prior work on axisymmetric bars where material lines have been assumed to remain normal to the axis, resulting in a second-gradient model which is formally similar to the asymptotically exact one but overestimates the modulus of the second-gradient term. In future work, it would be interesting to extend the present work and take into account this curvature effect in the derivation of second-gradient models coupled to physical fields, such as thin elastic biomembranes coupled to the concentration of chemical species (Deseri et al., 2008), or elastic membranes to an electric field (De Tommasi et al., 2013).

In this paper the model has been used to demonstrate the exceptional difference between the necking behavior of nonlinear elastic materials and elastic-plastic materials. For nonlinear elastic materials which have a maximum load under uniform tension, bifurcation into a necking mode occurs shortly beyond attainment of the maximum load and is highly unstable, snapping dynamically under prescribed elongation. By contrast, the post-bifurcation response of an elastic-plastic material specimen displays stable behavior for a range of elongations beyond the maximum load. The extent of the stable range depends on the slenderness of the specimen. Very slender specimens such as long wires or thin sheets lose stability almost immediately upon attaining the maximum load with all plastic deformation localized at a neck which shrinks to zero cross-section.

The one-dimensional model facilitates analytical investigations of necking and localization. Coleman and Newman (1988) developed an earlier version of the model for the purpose of studying the drawing process of polymer fibers' steady-state neck propagation in materials which undergo a local maximum load under uniaxial tension followed by strengthening with further stretching. The model holds promise for advancing the understanding of other important necking phenomena. If it can be extended to encompass rate-dependent material behavior, it will provide a valuable extension of the long-wavelength approximation used by Hutchinson and Neale (1977) and Ghosh (1977) to analyze the significant influence of material strain-rate sensitivity on necking in the tensile test. Rate-sensitivity becomes increasingly important at higher temperatures and its influence on necking is not well characterized. The one-dimensional model should be well suited for investigating phenomena such as the occurrence of multiple necks.

Another area of potential application for the one-dimensional model is sheet metal necking. The Marciniak-Kuczyński model (Marciniak and Kuczyński, 1967) is a long-wavelength approximation for predicting the onset of necking in sheets subject to general combinations of in-plane stretch. The outcome of such analyses is the forming limit diagram used to design metal forming processes. By including the influence of the gradients of the strain, the one-dimensional model would predict detailed spatial aspects of neck development in the sheet which the long-wavelength approximation does not provide. In the range of imposed in-plane strains in which the localized neck is not perpendicular to the maximum principle strain, the one-dimensional model would have to be extended to include shearing strains parallel to the localization band. For sheet metal necking and other necking problems, it is straightforward to account for initial imperfections in the model. 


\section{Appendix A. Manipulation of integrals}

Numerical integration of the integrals in equations (3.13-3.14) is required to generate the curves in Section 3. These integrals are ill-conditioned at their lower limit when $\lambda_{R} \cong \lambda_{R}^{*}$ defined in (3.15), which occurs for all but stocky blocks. In the following, the integrals are manipulated into a form that extracts the analytical dependence on $\lambda_{R}$ as $\lambda_{R} \rightarrow \lambda_{R}^{*}$ resulting in integrals which are well-condition for numerical integration. Consider (3.14):

$$
\int_{\lambda_{R}}^{\lambda_{L}} \frac{d \lambda^{\prime}}{\sqrt{R\left(\lambda^{\prime}\right)}}=\frac{L}{h}
$$

where

$$
R(\lambda)=\frac{2 \Delta^{2}\left(\lambda_{L}, \lambda_{R}, \lambda\right)}{b(\lambda)}=\frac{2}{b(\lambda)}\left(w(\lambda)-\tilde{w}\left(\lambda_{R}, \lambda_{L}, \lambda\right)\right)
$$

and

$$
\tilde{w}\left(\lambda_{R}, \lambda_{L}, \lambda\right)=\frac{w\left(\lambda_{R}\right)\left(\lambda_{L}-\lambda\right)+w\left(\lambda_{L}\right)\left(\lambda-\lambda_{R}\right)}{\lambda_{L}-\lambda_{R}} .
$$

The Taylor expansion of $R(\lambda)$ at $\lambda=\lambda_{R}$ reads $R(\lambda)=A\left(\lambda-\lambda_{R}\right)+B\left(\lambda-\lambda_{R}\right)^{2}+\cdots$, where

$$
\begin{aligned}
& A=R^{\prime}\left(\lambda_{R}\right)=\frac{2}{b\left(\lambda_{R}\right)}\left[w^{\prime}\left(\lambda_{R}\right)-\frac{w\left(\lambda_{L}\right)-w\left(\lambda_{R}\right)}{\lambda_{L}-\lambda_{R}}\right] \\
& B=\frac{1}{2} R^{\prime \prime}\left(\lambda_{R}\right)=-\frac{2 b^{\prime}\left(\lambda_{R}\right)}{b\left(\lambda_{R}\right)^{2}}\left[w^{\prime}\left(\lambda_{R}\right)-\frac{w\left(\lambda_{L}\right)-w\left(\lambda_{R}\right)}{\lambda_{L}-\lambda_{R}}\right]+\frac{1}{b\left(\lambda_{R}\right)} w^{\prime \prime}\left(\lambda_{R}\right)
\end{aligned}
$$

and $(\cdot)^{\prime}=\mathrm{d}(\cdot) / \mathrm{d} \lambda$. At $\lambda=\lambda_{L}$, the Taylor expansion reads $R(\lambda)=C\left(\lambda_{L}-\lambda\right)+\cdots$, where

$$
C=R^{\prime}\left(\lambda_{L}\right)=\frac{2}{b\left(\lambda_{L}\right)}\left[\frac{w\left(\lambda_{L}\right)-w\left(\lambda_{R}\right)}{\lambda_{L}-\lambda_{R}}-w^{\prime}\left(\lambda_{L}\right)\right]
$$

For the functions $w(\lambda)$ of interest, $A \geq 0, B>0$ and $C>0$, but $A \rightarrow 0$ as $\lambda_{R} \rightarrow \lambda_{R}^{*}$ by definition of $\lambda_{R}^{*}$. As a result, the integrand in (A.1) is not integrable at the lower limit as $\lambda_{R} \rightarrow \lambda_{R}^{*}$, although it is integrable at $\lambda_{L}$. Divide the integral into two contributions: $\lambda_{R} \leq \lambda \leq \lambda_{M}$ and $\lambda_{M} \leq \lambda \leq \lambda_{L}$ with $\lambda_{M}=\left(\lambda_{R}+\lambda_{L}\right) / 2$. Let $f(\lambda)=A\left(\lambda-\lambda_{R}\right)+B\left(\lambda-\lambda_{R}\right)^{2}$ and rewrite (A.1) as

$$
\int_{\lambda_{R}}^{\lambda_{L}} \frac{\mathrm{d} \lambda^{\prime}}{\sqrt{R\left(\lambda^{\prime}\right)}}=\int_{\lambda_{R}}^{\lambda_{M}}\left(\frac{1}{\sqrt{R\left(\lambda^{\prime}\right)}}-\frac{1}{\sqrt{f\left(\lambda^{\prime}\right)}}\right) \mathrm{d} \lambda^{\prime}+\int_{\lambda_{R}}^{\lambda_{M}} \frac{\mathrm{d} \lambda^{\prime}}{\sqrt{f\left(\lambda^{\prime}\right)}}+\int_{\lambda_{M}}^{\lambda_{L}} \frac{\mathrm{d} \lambda^{\prime}}{\sqrt{R\left(\lambda^{\prime}\right)}} .
$$

The first and third integrals above are bounded as $\lambda_{R} \rightarrow \lambda_{R}^{*}$ and can be integrated numerically with due regard for the end-point singularity. The second integral is given by

$$
\int_{\lambda_{R}}^{\lambda_{M}} \frac{\mathrm{d} \lambda^{\prime}}{\sqrt{f\left(\lambda^{\prime}\right)}}=\frac{2}{\sqrt{B}} \sinh ^{-1}\left(\sqrt{\frac{B\left(\lambda_{M}-\lambda_{R}\right)}{A}}\right)
$$

Manipulation of the other integrals such as (3.13) and those appearing below equation (3.14) is also required and similar.

\section{Appendix B. Numerical method for solving the necking problem with linear elastic unloading}

The energy functional governing the block is $W_{\mathrm{ep}}+E_{\mathrm{p}}$, with $W_{\mathrm{ep}}$ given by (4.3). We start by deriving the equation of equilibrium in weak form by canceling the first variation of $W_{\mathrm{ep}}+E_{\mathrm{p}}$ for any virtual increment $\hat{\lambda}$ of the stretch,

$$
0=h \int_{0}^{L}\left[w^{\prime} \hat{\lambda}+\frac{h^{2}}{2} b^{\prime} \lambda^{\prime 2} \hat{\lambda}+h^{2} b \lambda^{\prime} \hat{\lambda}^{\prime}+\alpha \frac{E}{\lambda_{\mathrm{u}}^{2}}\left(\lambda-\lambda_{\mathrm{u}}\right) \hat{\lambda}-n \hat{\lambda}\right] \mathrm{d} X .
$$


Integrating by parts, one could find an equation which extends the o.d.e. (3.8) and accounts for unloading, but it is preferable to continue working with the weak form.

The simulation solves for increments $\dot{\lambda}(X)$ of the stretch, in terms of increments $\dot{n}$ of the load, with $\lambda(X)$ known from the previous step. The incremental equation of equilibrium is obtained by linearizing the above weak form (second variation of the energy),

$$
0=h \int_{0}^{L}\left[\left(w^{\prime \prime}+\frac{h^{2}}{2} b^{\prime \prime} \lambda^{\prime 2}+\alpha \frac{E}{\lambda_{\mathrm{u}}^{2}}\right) \dot{\lambda} \hat{\lambda}+h^{2} b^{\prime} \lambda^{\prime} \frac{\mathrm{d}(\dot{\lambda} \hat{\lambda})}{\mathrm{d} X}+h^{2} b \dot{\lambda}^{\prime} \hat{\lambda}^{\prime}-\dot{n} \hat{\lambda}\right] \mathrm{d} X
$$

Integrating by parts and dropping the boundary term, we have

$$
0=h \int_{0}^{L}\left[\left(w^{\prime \prime}+\frac{h^{2}}{2} b^{\prime \prime} \lambda^{\prime 2}-h^{2} \frac{\mathrm{d}\left(b^{\prime} \lambda^{\prime}\right)}{\mathrm{d} X}+\alpha \frac{E}{\lambda_{\mathrm{u}}^{2}}\right) \dot{\lambda} \hat{\lambda}+h^{2} b \dot{\lambda}^{\prime} \hat{\lambda}^{\prime}-\dot{n} \hat{\lambda}\right] \mathrm{d} X
$$

which can be simplified as

$$
0=h \int_{0}^{L}\left[\left(w^{\prime \prime}-\frac{h^{2}}{2} b^{\prime \prime} \lambda^{\prime 2}-h^{2} b^{\prime} \lambda^{\prime \prime}+\alpha \frac{E}{\lambda_{\mathrm{u}}^{2}}\right) \dot{\lambda} \hat{\lambda}+h^{2} b \dot{\lambda}^{\prime} \hat{\lambda}^{\prime}-\dot{n} \hat{\lambda}\right] \mathrm{d} X
$$

Renaming the increment $\hat{\lambda}$ as $\hat{\dot{\lambda}}$, this equation can be seen as the condition of stationarity of a potential

$$
F(\dot{\lambda})=\int_{0}^{L}\left[\left(w^{\prime \prime}-\frac{h^{2}}{2} b^{\prime \prime} \lambda^{\prime 2}-h^{2} b^{\prime} \lambda^{\prime \prime}+\alpha \frac{E}{\lambda_{\mathrm{u}}^{2}}\right) \frac{\dot{\lambda}^{2}}{2}+h^{2} b \frac{\left(\dot{\lambda}^{\prime}\right)^{2}}{2}-\dot{n} \dot{\lambda}\right] \mathrm{d} X .
$$

In the simulation, suppose $\lambda(X)$ is known in the current state and the current location of the boundary between the loading and unloading regions is $X=X_{\mathrm{u}}$ with $\lambda_{\mathrm{u}}(X)$ known for $X \geq X_{\mathrm{u}}$ from previous iterations (the stretch $\lambda_{\mathrm{u}}(X)$ at which unloading starts at a given point $X$ is an essential unknown of the simulation). In equation (B.1), we then take $\alpha=1$ for $X \geq X_{\mathrm{u}}$ and $\alpha=0$ for $X<X_{\mathrm{u}}$. The next increment of stretch $\dot{\lambda}(X)$ is found by rendering the quadratic functional $F(\dot{\lambda})$ stationary, subject to prescribed negative increments of $\dot{n}$ : this is equivalent to solving the linearized equilibrium. The rapid spread of the unloading region requires special attention as will be discussed shortly.

To generate numerical results a simple finite element-like scheme is adopted. The interval $0 \leq X \leq L$ is divided into $M$ equal length segments and a piecewise linear approximation to $\lambda(X)$ is sought in terms of its nodal values $\lambda_{i}$ at the ends of the segments $X_{i}, i=0, M$. At the current stage of deformation, the contributions to the coefficients in (B.1) depend on $\lambda(X), \lambda^{\prime}(X)$ and $\lambda^{\prime \prime}(X)$. These are evaluated at the interval mid-points using standard finite difference expressions, as are the expressions for the increments $\dot{\lambda}$ and $\dot{\lambda}^{\prime}$. Summing the mid-point contributions to (B.1) gives the algebraic approximation

$$
F=\sum_{i, j=0}^{M} C_{i j} \dot{\lambda}_{i} \dot{\lambda}_{j}-\dot{n} \sum_{i=0}^{M} c_{i} \dot{\lambda}_{i}
$$

The boundary conditions at the ends are natural, meaning that no constraint on $\dot{\lambda}_{0}$ or $\dot{\lambda}_{M}$ is required in rendering $F$ stationary.

In principle, one could use $\dot{n}$ as the prescribed control parameter in a step-by-step incremental solution procedure, but given the exceptionally strong dependence of $X_{\mathrm{u}}$ on $n$ seen in figure $6 \mathrm{~b}$, it is preferable to prescribe increments of the unloading boundary $\dot{X}_{\mathrm{u}}$ and compute the associated increment $\dot{n}$. This procedure has been adopted here. Specifically, the computation was devised to shift the unloading boundary by exactly one interval, say from $X_{k+1}$ to $X_{k}$, and $\dot{n}$ is chosen to satisfy the condition $\dot{\lambda}_{k}=0$ at the end of the step. With square brackets denoting matrices and vectors, the stationarity condition for $F$ in (B.2) is $[\dot{\lambda}]=\frac{\dot{n}}{2}[C]^{-1} \cdot[c]$. If $[C]$ and $[c]$ are evaluated at the start of the incremental step (and are therefore independent of $[\dot{\lambda}]$ ), $\dot{\lambda}_{k}$ is proportional to $\dot{n}$ by the stationarity condition for $F$, implying that $\dot{\lambda}_{k}=0$ cannot select the value of $\dot{n}$. This dictates that $[C]$ and $[c]$ should be evaluated using stretch values at the end of the step, $[\lambda+\dot{\lambda}]$ : an 
iterative procedure is required to obtain $\dot{n}$ for each incremental advance of the unloading boundary. When $\dot{n}$ and $[\dot{\lambda}]$ are obtained such that $\dot{\lambda}_{k}=0$, the stretch values are updated for the next unloading step and $\lambda_{\mathrm{u}}\left(X_{k}\right)$ is assigned. The numerical results plotted in Section 4 have been computed with $M=200$. Curves computed with $M=100$ are virtually indistinguishable from those presented in Section 4.

Antman, S. S., 1973. Nonuniqueness of equilibrium states for bars in tension. Journal of Mathematical Analysis and Applications $44(2), 333-349$.

Antman, S. S., Carbone, E. R., 1977. Shear and necking instabilities in nonlinear elasticity. Journal of Elasticity 7 (2), $125-151$. Bridgman, P. W., 1952. Studies in large plastic flow and fracture. Metallurgy and metallurgical engineering series. McGraw-Hill. Chen, W. H., 1971. Necking of a bar. International Journal of Solids and Structures 7 (7), 685-717.

Coleman, B. D., Newman, D. C., 1988. On the rheology of cold drawing. I. elastic materials. Journal of Polymer Science: Part B: Polymer Physics 26, 1801-1822.

Considère, A., 1885. Mémoire sur l'emploi du fer et de l'acier dans les constructions. Annales des Ponts et Chaussées, Série 6 $9,574-775$.

De Tommasi, D., Puglisi, G., Zurlo, G., 2013. Electromechanical instability and oscillating deformations in electroactive polymer films. Applied Physics Letters 102, 011903.

Deseri, L., Piccioni, M. D., Zurlo, G., 2008. Derivation of a new free energy for biological membranes. Continuum Mechanics and Thermodynamics 20 (5), 255-273.

Doedel, E. J., Champneys, A. R., Fairgrieve, T. F., Kuznetsov, Y. A., Sandstede, B., Wang, X. J., 2007. AUTO-07p: continuation and bifurcation software for ordinary differential equations. See http://indy.cs.concordia.ca/auto/.

Ericksen, J. L., 1975. Equilibrium of bars. Journal of Elasticity 5 (3-4), 191-201.

Ghosh, A. K., 1977. Tensile instability and necking in materials with strain hardening and strain-rate hardening. Acta Metallurgica $12(25), 1413-1424$.

Hill, R., 1961. Bifurcation and uniqueness in nonlinear mechanics of continua. In: Problems in continuum Mechanics (Muskhelishvili Anniversary Volume). SIAM, Philadelphia (PA), pp. 155-164.

Hill, R., Hutchinson, J. W., 1975. Bifurcation phenomena in the plane tension test. Journal of the Mechanics and Physics of Solids $23(4-5), 239-264$.

Hutchinson, J. W., Miles, J. P., 1974. Bifurcation analysis of the onset of necking in an elastic/plastic cylinder under uniaxial tension. Journal of the Mechanics and Physics of Solids 22, 61-71.

Hutchinson, J. W., Neale, K. W., 1977. Influence of strain-rate sensitivity on necking under uniaxial tension. Acta Metallurgica $25,839-846$.

Koiter, W. T., 1965. On the stability of elastic equilibrium. Ph.D. thesis, Delft University, Holland.

Marciniak, K., Kuczyński, K., 1967. Limit strains in the process of stretch forming sheet metal. International Journal of Mechanical Sciences 9, 609-620.

Mielke, A., 1991. Hamiltonian and Lagrangian flows on center manifolds, with application to elliptic variational problems. Vol. 1489 of Lecture notes in mathematics. Springer-Verlag.

Needleman, A., 1972. A numerical study of necking in circular cylindrical bar. Journal of the Mechanics and Physics of Solids $20(2), 111-127$.

Norris, D. M., Moran, B., Scudder, J. K., Quiñones, D. F., 1978. A computer simulation of the tension test. Journal of the Mechanics and Physics of Solids 26 (1), 1-19.

Owen, N., 1987. Existence and stability of necking deformations for nonlinearly elastic rods. Archive for Rational Mechanics and Analysis 98 (4), 357-383.

Scherzinger, W., Triantafyllidis, N., 1998. Asymptotic analysis of stability for prismatic solids under axial loads. Journal of the Mechanics and Physics of Solids 46 (6), 955-1007.

Sivaloganathan, J., Spector, S. J., 2011. On the stability of incompressible elastic cylinders in uniaxial extension. Journal of Elasticity $105(1-2), 313-330$.

Triantafyllidis, N., Scherzinger, W. M., Huang, H.-J., 2007. Post-bifurcation equilibria in the plane-strain test of a hyperelastic rectangular block. International Journal of Solids and Structures 44 (11-12), 3700-3719.

van der Heijden, A. M. A., 2008. W. T. Koiter's Elastic stability of solids and structures. Cambridge University Press. 